\title{
On Optimal Investment in a Reinsurance Context with a Point Process Market Model
}

\author{
Enrico Edoli* and Wolfgang J. Runggaldier ${ }^{\dagger}$ \\ Department of Pure and Applied Mathematics, University of Padova, \\ Via Trieste, 63, I-35121 Padova, Italy \\ e-mail: enricoedoli@gmail.com,runggal@math.unipd.it
}

\begin{abstract}
We study an insurance model where the risk can be controlled by reinsurance and investment in the financial market. We consider a finite planning horizon where the timing of the events, namely the arrivals of a claim and the change of the price of the underlying asset(s), corresponds to a Poisson point process. The objective is the maximization of the expected total utility and this leads to a nonstandard stochastic control problem with a possibly unbounded number of discrete random time points over the given finite planning horizon. Exploiting the contraction property of an appropriate dynamic programming operator, we obtain a value-iteration type algorithm to compute the optimal value and strategy and derive its speed of convergence. Following Schäl(2004) we consider also the specific case of exponential utility functions whereby negative values of the risk process are penalized thus combining features of ruin minimization and utility maximization. For this case we are able to derive an explicit solution. Results of numerical computations are also reported.
\end{abstract}

JEL: G22, G11

MSC: 91B30, 93E20, 90C40, 90C39

PACS: IM10, IM52

Keywords: Reinsurance and investment, expected utility maximization, ruin minimization, value iteration.

\section{Introduction}

We study an insurance model where the risk/reserve process can be controlled by reinsurance and investment in the financial market. In this context the usual aim is to minimize the ruin probability over a given horizon. Inspired by Schäl(2004), in this paper we try to combine features of ruin probability minimization with expected utility maximization by formulating an objective function that consists in the maximization of the expected cumulative exponential utility from the risk/reserve process over a given planning horizon; by maximizing expected exponential

${ }^{*}$ Present affiliation: Finalyst s.a.s., via Trentino 28, Campolongo Maggiore (VE)

${ }^{\dagger}$ Corresponding author. Tel.: +39-049-8271454, Fax: +39-049-8271499 
utility one penalizes in fact negative values of the risk/reserve process. An analogous purpose appears partly in the recent paper Bai and Guo(2008), where the authors study the problem of expected exponential utility of terminal wealth as well as that of the minimization of the ruin probability for a reinsurance and investment model, but the two problems are studied separately with one of the objectives being also to investigate the relationship between the two criteria.

Control problems for risk/reserve processes are commonly formulated in continuous time. Schäl(2004) presents a formulation of the problem where events (arrivals of claims and asset price changes) occur at discrete points in time that may be deterministic or random, but their total number is fixed. This implies that in the case of random time points the horizon is random as well. We feel that a more natural way to formulate the problem in case of random time points is to consider a given fixed time horizon so that it is the number of event times that becomes random and this makes the problem nonstandard. With random event times it is reasonable to assume that also the control decisions (level of reinsurance and investment) correspond to these random discrete time points. This is consistent both with the realistic impossibility of changing the reinsurance contract continuously as well as with the fact that trading occurs generally only at the times when quotes are updated in the market. Notice that this problem formulation can be seen equivalently in discrete or continuous time. In fact, although the processes to be considered are defined in discrete time as sequences of claims, price changes and controls, one has that by means of piecewise constant interpolations, which for the controls have to be left continuous to make them predictable, they can also be viewed as continuous time processes. In the problem formulation below we shall rather naturally opt for the continuous time setup.

The problem results thus in a stochastic control problem that can be seen in discrete or continuous time. A traditional approach in a continuous time setting is via the Hamilton-JacobiBellman (HJB) theory. To this effect see e.g. Schmidli(2002) and Luo et al.(2008) in an insurance context where the criterion is the minimization of the ruin probability and, more recently, Bai and $\operatorname{Guo}(2008)$ where both minimization of ruin probability as well as maximization of expected exponential utility of terminal wealth are considered (for this latter criterion see also Cao and Wan(2009)). The HJB theory, which traditionally was derived for diffusion-type processes, can in fact be applied to discontinuous processes as well (for general references see e.g. Hipp(2004), Schmidli(2008)). The HJB approach requires however appropriate regularity of the value function as well as other properties that are not always easy to verify; furthermore, analytic solutions are difficult to come by.

In the present paper we focus on value iteration that does not require these regularity assumptions and in certain cases (see Section 4 below) allows for an analytic solution. In those cases where an analytic solution may be difficult to obtain, it allows to compute the optimal values and control to any degree of accuracy. In fact, the optimal value turns out to be the fixed point of a suitable contraction operator. The solution involves thus the determination of this fixed point that, thanks to the contraction property of the operator, can arbitrarily closely be approximated by iterating sufficiently often this operator. In Section 4 it will be shown that, by choosing an exponential utility function that fulfills our purpose of combining features of ruin minimization and utility maximization, it is possible to obtain a semianalytic solution to the problem in the sense that the solution to the fixed point problem can be expressed in terms of two Volterra integral equations that can be solved explicitly; the controls are then determined numerically.

Value iteration is a natural approach to problems formulated in discrete time and is traditionally studied for the case of deterministic time points. Inspired by results in Kirch and Runggaldier(2004), where hedging problems are considered, we extend it here to the case of discrete random event times. In the context of portfolio optimization an analogous approach can be found in Bäuerle and Rieder(2009) and DiMasi et al.(2006) (somewhat related are also the 
papers Pham and Tankov(2008) and Pham and Tankov(2009)).

The structure of the paper is as follows. In the next Section 2 we describe the model and formulate the objective. In Section 3 we present the value iteration approach for general utility functions. In Section 4 we discuss the solution of our problem via value iteration by presenting first a semianalytic solution for the case of exponential utility functions and then discussing numerical implementations of value iteration in the general case. Results of numerical calculations are also reported.

\section{Model and objective}

Choosing a continuous time setup we consider a risk/reserve process of a company (in our context we shall call it the wealth process) that results from deterministic premium and stochastic claim payments as well as from investment in the financial market.

The stochastic elements that affect the evolution of the risk process are the timing and size of the claims as well as the evolution of the prices of the assets in which the company is investing. We allow for two possibilities to intervene in (control) the evolution of the risk process: reinsurance and investment.

Given a time horizon, the objective is to choose over time the reinsurance level and the amount of investment in order to maximize the expected cumulative utility.

Claims occur at random points in time and also their sizes are random, while asset price evolutions are usually modeled as continuous time processes. Noticing that, on small time scales, prices actually change at discrete random time points and vary by tick size, in our model we let also asset prices change only at discrete random times with their sizes being random as well. For simplicity and without loss of generality we shall assume that there is only one risky asset to invest in. We furthermore assume that the sizes of both the claims and the prices are i.i.d. with a positive probability mass at the value zero. This will allow us to consider the timing of the events (arrival of a claim and change of the price) to be triggered by a same counting process that we shall assume to be of the Poisson type. If then at an event time the size of a claim results being equal to zero, it means that at that time only a price change may occur and viceversa. Since between event times the situation for the company does not change, we shall determine the controls (level of reinsurance and investment) only at event times.

\subsection{Specific description of the model}

\subsubsection{Claims and price processes.}

Let:

- $[0, T]$ be a given planning horizon, $T<+\infty$;

- $N_{t}$ : a Poisson process with deterministic intensity $\lambda_{t}=\lambda$ that determines the timing of the events (arrivals of claims and price variation of the asset to invest in);

- $T_{i}$ : random time of occurrence of the $i$-th event:

$$
T_{i}=\inf \left\{t \geq 0 \mid N_{t}=i\right\}
$$

$T_{i+1}-T_{i}$ is exponentially distributed with parameter $\lambda$ independently of $i$.

- $Y_{T_{i}}$ : claim (payment) at $T_{i}$. The sequence $\left\{Y_{T_{i}}\right\}$ is supposed to be i.i.d. with a point mass at 0 . For simplicity, and without loss of generality, assume just two values for the claim sizes, namely $Y_{T_{i}} \in\{0, \bar{y}\}$ with $\mathbb{P}\left[Y_{T_{i}}=\bar{y}\right]=\bar{p}$; 
- $S_{t}$ : the discounted price process of the risky asset. For simplicity and without loss of generality we consider only one asset to invest in. We assume that prices change only at the event times $T_{i}$ according to

$$
\frac{S_{T_{i}}-S_{T_{i}}-}{S_{T_{i}-}}=e^{W_{T_{i}}-1}
$$

where $W_{T_{i}}$ is an i.i.d. sequence taking values in $\in[\underline{w}, \bar{w}]$ with $\underline{w}<0<\bar{w}$ and a point mass at 0 . Notice that, according to the given dynamics, prices can increase or decrease but, given that $e^{W_{T_{i}}-1}>-1$, they remain always positive. For simplicity, we let $W_{T_{i}}$ take only three representative values, i.e. $W_{T_{i}} \in\{-d, 0, u\}$ with probabilities $p_{1}, p_{2}, p_{3}$ respectively and with $d, u>0$.

Instead of considering the above random sequences, we may also work with continuous time processes. To the sequences $Y_{T_{i}}, W_{T_{i}}$ we may then equivalently associate the continuous time processes $Y_{t}, W_{t}$ considering them to be piecewise constant interpolations of $\left\{Y_{T_{n}}\right\}_{n \geq 0}$ and $\left\{W_{T_{n}}\right\}_{n \geq 0}$ respectively. The dynamics of $S_{t}$ is then given by:

$$
d S_{t}=S_{t^{-}}\left(e^{W_{t}}-1\right) d N_{t}
$$

i.e.

$$
S_{t}=S_{0}+\sum_{n=1}^{N_{t}} S_{T_{n}^{-}}\left(e^{W_{T_{n}}}-1\right)
$$

- $N_{t}, Y_{t}, W_{t}$ are supposed to be independent. The independence between $Y$ and $W$ can easily be relaxed as in all our calculations only their joint distribution is involved; also independence with $N$ can be relaxed but then the calculations get more involved.

- Let $(\Omega, \mathcal{F}, \mathbb{P})$ be the probability space on which all the stochastic processes to be considered are defined, and let $\left\{\mathcal{F}_{t}\right\}_{t \geqslant 0} \subseteq \mathcal{F}$ be the smallest filtration that makes the processes $N_{t}$, $Y_{t}, W_{t}$ measurable.

\subsubsection{Controls: reinsurance and investment.}

Control decisions are supposed to correspond to the event times and they have to be determined on the basis of information prior to the generic $T_{i}$, so they have to be left continuous ("predictable").

We consider a proportional reinsurance: for $b_{T_{i}} \in[0,1]$, the part of the claim paid by the company is $h\left(b_{T_{i}}, Y_{T_{i}}\right)=b_{T_{i}} \cdot Y_{T_{i}}$. If $b=0$ all the risk is reinsured, if $b=1$ there is no reinsurance.

The control $\delta_{T_{i}}$ represents the amount invested in the risky asset at the event time $T_{i}$. We assume also that the company is not allowed to be indebted beyond a certain level, nor to invest beyond a certain level: this leads to having $\delta_{T_{i}} \in\left[-C_{1}, C_{2}\right]$ for all $T_{i}$ for some constants $C_{1}, C_{2}>0$. We shortly write $\phi_{T_{i}}=\left(b_{T_{i}}, \delta_{T_{i}}\right)$ for the control in the interval $\left(T_{i-1}, T_{i}\right]$. and

$$
U=[0,1] \times\left[-C_{1}, C_{2}\right]
$$

for the compact control space.

\subsubsection{Premium Rates.}

Let $c$ be the premium rate paid by the customer to the company, fixed in the contract. To obtain the reinsurance, the company must pay to the reinsurer another premium rate, depending 
on the reinsurance level $b$, so let $c(b)$ be the net income rate of the company. If $h(b, y)$ represents the part of the claim paid by the company $(h(0, y)=0)$, according to the expected value principle with safety loading $\theta$ of the reinsurer, $c(b)$ can be chosen as follows:

$$
c(b):=c-(1+\theta) \frac{\mathbb{E}[Y-h(b, Y)]}{\mathbb{E}[\Xi]}
$$

where $\Xi$ is the random time between two claims. Notice that, under proportional reinsurance, $c(b)$ is a continuous function of $b$ and, clearly, $c$ is chosen so that $c(b) \geq 0 \quad \forall b \in[0,1]$. Since $c(b)$ is increasing in $b$ one has $c(0) \leqslant c(b) \leqslant c(1)=c$ so it suffices to choose

$$
c=(1+\theta) \frac{\mathbb{E}[Y]}{\mathbb{E}[\Xi]}
$$

Condition (3) also guarantees that for any $b \in[0,1]$ one has $c(b) \geq 0$ and that, since $h(0, y)=0$, by taking $b=0$ (complete reinsurance) the net income rate is $c(b)=0$.

\subsubsection{The wealth/reserve}

Given an initial capital $X_{0}$, let $X_{t}$ be the discounted "risk/reserve process" at time $t \in[0, T]$ that takes into account:

- the net premium rate collected so far;

- the amount invested at time $t$ in the stock $S_{t}$

- the total claims paid by the company until time $t$

So the formula for $X_{t}$ is:

$$
X_{t}=X_{0}+\int_{0}^{t} c\left(b_{t}\right) d t+\sum_{n=1}^{N_{t}}\left[\delta_{T_{n}}\left(e^{W_{T_{n}}}-1\right)-h\left(b_{T_{n}}, Y_{T_{n}}\right)\right]
$$

Notice that $\delta_{T_{n}}$ denotes the monetary amount invested in the risky asset at the event time $T_{n}$ and the controls have been assumed to be left continuous (predictable) i.e. $\delta_{T_{n}}=\delta_{T_{n}-}$. Denoting then by $\alpha_{T_{n}}:=\frac{\delta_{T_{n}}}{S_{T_{n}-}}$ the (also left continuous) number of units invested in the risky asset at the event time $T_{n}$, from (1) we have for the generic term in the sum on the right hand side of (4) that

$$
\delta_{T_{n}}\left(e^{W_{T_{n}}}-1\right)=\delta_{T_{n}} \frac{S_{T_{n}}-S_{T_{n}-}}{S_{T_{n}-}}=\alpha_{T_{n}}\left(S_{T_{n}}-S_{T_{n}-}\right)
$$

and this gives the (self financing) increment of the investment in the financial market due to the change, from $S_{T_{n}-}$ to $S_{T_{n}}$, in the asset price at the event time $T_{n}$.

In differential form (4) becomes

$$
d X_{t}=c\left(b_{t}\right) d t+\left[\delta_{t}\left(e^{W_{t}}-1\right)-h\left(b_{t}, Y_{t}\right)\right] d N_{t}
$$

Notice that, while $b_{t}, \delta_{t}$ are left-continuous, $W_{t}, Y_{t}$ may be considered as right continuous.

We now have

Definition 1. The set $\Phi$ of admissible controls is

$$
\Phi=\left\{\phi=(b, \delta) \quad \text { s.t. } \quad \phi \text { is a U-valued process, constant in }\left(T_{i-1}, T_{i}\right], i=1, \ldots, N_{T}\right\}
$$


In what follows we shall denote by $X_{t}^{\phi}$ the solution of (4) corresponding to the control strategy $\phi_{t}=\left(b_{t}, \delta_{t}\right)$.

Remark 2. The optimal controls will later on be determined as feedback controls of the form $\phi=\phi(t, x)=(b(t, x), \delta(t, x))$ and, given our assumptions, will be continuous functions of their arguments. In what follows, whenever it applies, we will thus implicitly consider the controls to be of the feedback form.

Notice that, since $c\left(b_{t}\right) \geqslant 0$, by choosing $b_{t} \equiv 0 \equiv \delta_{t}$ (no investment and complete reinsurance), the value of $X_{t}$ cannot decrease. This is a trivial case that we do not consider, but it ensures that $\Phi \neq \emptyset$.

\subsubsection{Value Function and The Objective.}

We want to maximize the expected "cumulative utility" from our wealth process over the given horizon $[0, T]$.

Although later on we shall more specifically consider exponential utility functions that, as already mentioned, allow to combine features of classical ruin minimization and utility maximization, we formulate here our objective for general utility functions.

Given a ("running") utility function $g: \mathbb{R} \rightarrow \mathbb{R}$ and a ("terminal") utility function $G: \mathbb{R} \rightarrow \mathbb{R}$, consider for $\phi \in \Phi$ the following Value Function:

$$
V^{\phi}(t, x):=\mathbf{1}_{\{t \leqslant T\}} \mathbb{E}_{t, x}^{\phi}\left[\sum_{k=N_{t}+1}^{N_{T}} g\left(X_{T_{k}}^{\phi}\right)+G\left(X_{T}^{\phi}\right)\right]
$$

that represents the total/cumulative expected utility starting from time $t$ with capital $X_{t}=x$ and using the control $\phi \in \Phi$ (notice that $\mathbb{E}_{t, x}^{\phi}$ is the mean under the conditional probability $\mathbb{P}\left[A \mid \mathcal{F}_{t}\right], A \in \mathcal{F}$, taking into account that $X_{t}$ is a Markov Process).

Notation 3. Henceforth we shall often use the notation

$$
V^{\phi}\left(T_{n}, x\right):=\mathbf{1}_{\left\{T_{n} \leqslant T\right\}} \mathbb{E}_{T_{n}, x}^{\phi}\left[\sum_{k=n+1}^{N_{T}} g\left(X_{T_{k}}^{\phi}\right)+G\left(X_{T}^{\phi}\right)\right]
$$

with the following meaning: having observed the jump time $T_{n}$ and the value $X_{T_{n}}=x, V^{\phi}\left(T_{n}, x\right)$ represents the total expected utility starting from time $T_{n}$ with capital $x$. Formally, we may consider $V^{\phi}\left(T_{n}, x\right)$ as the function $V^{\phi}(t, x)$ evaluated at $t=T_{n}$ for the given $X_{T_{n}}=x$.

Recalling that the control decisions (reinsurance and investment level) correspond to event times, we use the shorthand notation $b_{h}=b_{T_{h}}, \delta_{h}=\delta_{T_{h}}$ for the controls to be applied in the interval $\left(T_{h-1}, T_{h}\right]$. Analogously we shall also put $Y_{h}:=Y_{T_{h}}, W_{h}:=W_{T_{h}}$. Furthermore, letting

$$
Z_{h+1}:=T_{h+1}-T_{h} \quad h \geqslant 0
$$

which are i.i.d. distributed according to a random variable $Z$ that has a negative exponential distribution with parameter $\lambda$, we have (see (4)) that at the generic jump time $T_{k}$ the value of $X_{T_{k}}^{\phi}$ is

$$
X_{T_{k}}^{\phi}=X_{0}+\sum_{h=1}^{k}\left[c\left(b_{h}\right) Z_{h}-h\left(b_{h}, Y_{h}\right)+\delta_{h}\left(e^{W_{h}}-1\right)\right]
$$


Noticing that, being $Z$ is a continuous random variable, we are allowed to write $\mathbf{1}_{\{t+Z \leqslant T\}}=$ $\mathbf{1}_{\{t+Z<T\}}$ a.s., define the random set of integers

$$
I_{n}(\omega):=\left\{k \in \mathbb{N} \mid \sum_{h=1}^{k} Z_{n+h}(\omega)<T-T_{n}\right\}
$$

We then have the following explicit formula for $V^{\phi}\left(T_{n}, x\right)$ :

$$
\begin{aligned}
& V^{\phi}\left(T_{n}, x\right) \\
& =\mathbf{1}_{\left\{T_{n} \leq T\right\}} \mathbb{E}^{\phi}\left[\sum_{k \in I_{n}} g\left(x+\sum_{h=1}^{k}\left[c\left(b_{n+h}\right) Z_{n+h}-h\left(b_{n+h}, Y_{n+h}\right)+\delta_{n+h}\left(e^{W_{n+h}}-1\right)\right]\right)\right. \\
& + \\
& +G\left(x+\sum_{h \in I_{n}}\left[c\left(b_{n+h}\right) Z_{n+h}-h\left(b_{n+h}, Y_{n+h}\right)+\delta_{n+h}\left(e^{W_{n+h}}-1\right)\right]\right. \\
& \left.\left.+c\left(b_{N_{T}+1}\right)\left(T-T_{N_{T}}\right)\right)\right]
\end{aligned}
$$

where the expectation is with respect to the joint distribution of the independent random variables $\left(Z_{n+h}, Y_{n+h}, W_{n+h}\right)$ and we have used the fact that the controls are predictable (left continuous) so that, see Definition $1, \phi_{n}=\left(b_{n}, \delta_{n}\right)$ is the control on the interval $\left(T_{n-1}, T_{n}\right]$ to be determined in $T_{n-1}$. For this reason we also write $b_{N_{T}+1}$ for the reinsurance ratio to be applied in $\left(T_{N_{T}}, T\right]$.

\subsubsection{The Problem.}

We want to maximize the total expected utility, i.e. determine $\phi^{*} \in \Phi$ s.t.

$$
V^{*}\left(T_{n}, x\right):=V^{\phi^{*}}\left(T_{n}, x\right)=\sup _{\phi \in \Phi} V^{\phi}\left(T_{n}, x\right)
$$

In particular, we are interested in $V^{*}\left(0, X_{0}\right)$.

Remark 4. For exponential utility functions that (see Section 4, in particular 4.1) we shall take of the form $g(x)=1-\gamma e^{-\beta x}, G(x)=1-\mu e^{-\beta x}$, we may interpret our objective function also as aiming at maximizing expected exponential utility from terminal wealth while keeping as low as possible the probability of occurrence of negative values of the risk process before the terminal time horizon. (In this context see also Bai and Guo(2008)).

\subsubsection{Possible approaches}

\section{Hamilton-Jacobi-Bellman (HJB) approach}

Our problem has been formulated as a stochastic control problem in continuous time. As mentioned in the Introduction, a traditional approach to such problems is the Hamilton-JacobiBellman(HJB) approach (see Hipp(2004), Schmidli(2008)). For our specific problem we shall now mention the formal derivation of of the Hamilton-Jacobi-Bellman equation by following the usual approach based on the Principle of Optimality. Assuming that all the required regularity properties hold, by using the generalized Ito formula for jump-diffusion processes and the fact that, being $N_{t}$ a Poisson process with deterministic intensity $\lambda_{t} \equiv \lambda$, one has

$$
\mathbb{E}\left[\sum_{k=n+1}^{N_{T}} g\left(X_{T_{k}}^{\phi}\right)\right]=\mathbb{E}\left[\int_{T_{n+1}}^{T} g\left(X_{s^{-}}^{\phi}\right) d N_{s}\right]=\mathbb{E}\left[\int_{T_{n+1}}^{T} \lambda \cdot g\left(X_{s}^{\phi}\right) d s\right]
$$


the following equation for the optimal value function $V^{*}(t, x)$ can rather straightforwardly be obtained (details are in Edoli (2009))

$$
\begin{aligned}
\partial_{t} V^{*}(t, x)+g(x)+ & \sup _{(b, \delta) \in \Phi}\left\{c(b) \partial_{x} V^{*}(t, x)+\right. \\
& \left.+\lambda \mathbb{E}\left[V^{*}\left(t, x+\delta\left(e^{W}-1\right)-h(b, Y)\right)-V^{*}(t, x)\right]\right\}=0
\end{aligned}
$$

with terminal condition $V^{*}(T, x)=G(x)$.

To solve (10) one has first to take the supremum over $b$ and $\delta$ for a hypothetical $V^{*}$, thus obtaining $b$ and $\delta$ in terms of $V^{*}$ and its derivatives. Then one replaces these expressions in formula (10) to obtain a PDE. Finally, to make sure that the controls so obtained as well as $V^{*}$ are optimal, a "verification theorem" has to be invoked.

Although the HJB approach can be used under restrictive assumptions also for our problem setting, it turns out that Value Iteration is a much more convenient approach.

\section{Value Iteration}

One of the weaknesses of the classical HJB approach are the regularity requirements for the value function $V(t, x)$. Although existence and uniqueness can be obtained also under weaker assumptions, the determination of the optimal controls via HJB still requires the existence of the partial derivatives of the solution.

Valuer iteration, which is the approach that we are going to follow, allows to overcome these problems and to actually determine the optimal value and the optimal controls to any degree of accuracy. In fact, differentiability of the value function is not needed and its existence and uniqueness follow from the contraction property of a suitable operator on a metric space. While for general utility functions it allows one to obtain the control (reinsurance and investment) to any level of suboptimality, provided the operator is iterated sufficiently often, for exponential utility functions we shall also provide a full solution that can be explicitly obtained by combining analytical and numerical techniques.

\section{The Value Iteration Approach}

In this section we study the value iteration approach to solve the problem formulated in section 2. The specific approach that we are going to use is inspired by Kirch and Runggaldier(2004).

\subsection{Mappings/operators}

We first recall the value functions.

Definition 5. Let $\phi \in \Phi, x \in \mathbb{R}, T_{n} \in \mathbb{R}^{+}, n \in \mathbb{N}, g: \mathbb{R} \rightarrow \mathbb{R}, G: \mathbb{R} \rightarrow \mathbb{R}$.

Given the Notation 3, recall the following functions:

$$
\begin{aligned}
V^{\phi}\left(T_{n}, x\right) & :=\mathbf{1}_{\left\{T_{n} \leqslant T\right\}} \mathbb{E}_{T_{n}, x}^{\phi}\left[\sum_{k=n+1}^{N_{T}} g\left(X_{T_{k}}^{\phi}\right)+G\left(X_{T}^{\phi}\right)\right] \\
V^{*}\left(T_{n}, x\right) & :=\sup _{\phi \in \Phi} V^{\phi}\left(T_{n}, x\right) \\
\phi^{*}\left(T_{n}, x\right) & :=\arg \left(\sup _{\phi \in \Phi} V^{\phi}\left(T_{n}, x\right)\right)
\end{aligned}
$$


with the meaning that, if $n+1>N_{T}$, then $\sum_{k=n+1}^{N_{T}} g\left(X_{T_{k}}^{\phi}\right)=0$ and where $\phi^{*}$ is now given in feedback form (see Remark 2).

Assumption 6. The functions $g: \mathbb{R} \rightarrow \mathbb{R}$ and $G: \mathbb{R} \rightarrow \mathbb{R}$ are increasing, concave, and bounded (i.e. $|g(x)| \leqslant \mathcal{G}$ and $|G(x)| \leqslant \mathcal{G}$ for some positive constant $\mathcal{G}$ ).

Definition 7. We denote by $\mathcal{B}\left(\mathbb{R}^{+} \times \mathbb{R}\right)$ the space of bounded functions on $\mathbb{R}^{+} \times \mathbb{R}$ and by $\mathcal{C}_{\mathcal{B}}\left(\mathbb{R}^{+} \times \mathbb{R}\right)$ the space of continuous and bounded functions on $\mathbb{R}^{+} \times \mathbb{R}$ endowed with the supnorm (for details see Section 3.3).

Remark 8. Given the Assumption 6, $V^{\phi}\left(T_{n}, x\right)$ is increasing in $x$ and bounded; in fact $-\mathbb{E}\left[N_{T}\right]$. $\mathcal{G} \leqslant V^{\phi}\left(T_{n},-K\right) \leqslant V^{\phi}\left(T_{n}, x\right) \leqslant \mathbb{E}\left[N_{T}\right] \cdot \mathcal{G}, \quad \forall T_{n} \in[0, T]$ with $\mathbb{E}\left[N_{T}\right]=\lambda T$, hence $V^{\phi}\left(T_{n}, x\right) \in$ $\mathcal{B}\left(\mathbb{R}^{+} \times \mathbb{R}\right)$.

Now we define the two main mappings/operators.

Definition 9. Given $g, G: \mathbb{R} \rightarrow \mathbb{R}$ satisfying Assumption 6, for $\phi \in \Phi$ and $Z \sim \operatorname{Exp}(\lambda)$ for some $\lambda>0$, define the operators $T^{\phi}, T^{*}: \mathcal{B}\left(\mathbb{R}^{+} \times \mathbb{R}\right) \rightarrow \mathcal{B}\left(\mathbb{R}^{+} \times \mathbb{R}\right)$ by

$$
\begin{gathered}
\left(T^{\phi} v\right)(t, x):=\mathbb{E}_{t, x}^{\phi}\left[\mathbf{1}_{\{t+Z \leqslant T\}} g\left(X_{t+Z}^{\phi}\right)+v\left(t+Z, X_{t+Z}^{\phi}\right)+\right. \\
\left.+\mathbf{1}_{\{t \leqslant T<t+Z\}} G\left(X_{T}^{\phi}\right)\right]
\end{gathered}
$$

where we want to point out that, given $t$ and $X_{t}^{\phi}=x$, the dependence on $\phi$ of $X_{t+Z}^{\phi}$ as well as of $X_{T}^{\phi}$ for the event $t \leqslant T<t+Z$ is only through its value $\phi_{N_{t}}$. This justifies the next definition, namely

$$
\left(T^{*} v\right)(t, x):=\sup _{\phi_{N_{t}}=\left(b_{N_{t}}, \delta_{N_{t}}\right) \in U}\left(T^{\phi} v\right)(t, x)
$$

Again, we denote

$$
\phi^{*}(t, x)=\arg \left(\sup _{\phi_{N_{t}} \in U}\left(T^{\phi} v\right)(t, x)\right)
$$

assuming it exists (see Proposition 15 below).

Definition 10. Let $G: \mathbb{R} \rightarrow \mathbb{R}$ satisfy Assumption $6, \phi \in \Phi, x \in \mathbb{R}, t \in[0, T]$. We define the sequence $\left\{v_{m}^{\phi}(t, x)\right\}_{m \in \mathbb{N}} \subseteq \mathcal{B}\left(\mathbb{R}^{+} \times \mathbb{R}\right)$ as:

$$
\left\{\begin{array}{l}
v_{0}^{\phi}(t, x):=\mathbf{1}_{\{t \leqslant T\}} G(x+c(b)(T-t)) \\
v_{m}^{\phi}(t, x):=\left(T^{\phi} v_{m-1}^{\phi}\right)(t, x)
\end{array}\right.
$$

and the sequence $\left\{v_{m}^{*}(t, x)\right\}_{m \in \mathbb{N}} \subseteq \mathcal{B}\left(\mathbb{R}^{+} \times \mathbb{R}\right)$ as:

$$
\left\{\begin{array}{l}
v_{0}^{*}(t, x):=\mathbf{1}_{\{t \leqslant T\}} \sup _{b \in[0,1]} G(x+c(b)(T-t)) \\
v_{m}^{*}(t, x):=\left(T^{*} v_{m-1}^{*}\right)(t, x)
\end{array}\right.
$$

The next subsection shows the relations between the value functions and the operators. 


\subsection{Fixed point characterization of the value functions}

Theorem 11. Let $\phi \in \Phi, x \in \mathbb{R}, T_{n} \in[0, T], \lambda>0, Z \sim \operatorname{Exp}(\lambda)$. We have:

i)

$$
\begin{aligned}
V^{\phi}\left(T_{n}, x\right)= & \mathbb{E}_{T_{n}, x}^{\phi}\left[\mathbf{1}_{\left\{T_{n}+Z \leqslant T\right\}} g\left(X_{T_{n}+Z}^{\phi}\right)+V^{\phi}\left(T_{n}+Z, X_{T_{n}+Z}^{\phi}\right)+\right. \\
& \left.+\mathbf{1}_{\left\{T_{n} \leqslant T<T_{n}+Z\right\}} G\left(X_{T}^{\phi}\right)\right]= \\
= & \left(T^{\phi} V^{\phi}\right)\left(T_{n}, x\right) \\
V^{*}\left(T_{n}, x\right)= & \sup _{\phi_{T_{n}} \in \Phi}\left\{\mathbb { E } _ { T _ { n } , x } ^ { \phi } \left[\mathbf{1}_{\left\{T_{n}+Z \leqslant T\right\}} g\left(X_{T_{n}+Z}^{\phi}\right)+\right.\right. \\
& \left.\left.+V^{*}\left(T_{n}+Z, X_{T_{n}+Z}^{\phi}\right)+\mathbf{1}_{\left\{T_{n} \leqslant T<T_{n}+Z\right\}} G\left(X_{T}^{\phi}\right)\right]\right\}= \\
= & \left(T^{*} V^{*}\right)\left(T_{n}, x\right)
\end{aligned}
$$

Proof. Part (i) Given the representation of $X_{T_{k}}^{\phi}$ in (7) and that $Z_{n}$ are i.i.d. $\sim Z$ which is an exponential random variable with parameter $\lambda$, we have $T_{n+1}=T_{n}+Z_{n+1}$ and $\left\{T_{n}+Z \leqslant T\right\} \subset\left\{T_{n} \leqslant T\right\}$, i.e. $\mathbf{1}_{\left\{T_{n} \leqslant T\right\}} \mathbf{1}_{\left\{T_{n}+Z \leqslant T\right\}}=\mathbf{1}_{\left\{T_{n}+Z \leqslant T\right\}}$, the expression for $V^{\phi}\left(T_{n}, x\right)$ in (8) can be rewritten as

$$
\begin{aligned}
V^{\phi}\left(T_{n}, x\right)= & \mathbf{1}_{\left\{T_{n} \leq T\right\}} \mathbb{E}^{\phi}\left[\mathbf{1}_{\left\{T_{n}+Z_{n+1} \leq T\right\}}\left(g\left(X_{T_{n}+Z_{n+1}}^{\phi}\right)+\sum_{h \in I_{n+1}} g\left(X_{h}^{\phi}\right)\right)+G\left(X_{T}^{\phi}\right)\right]= \\
= & \mathbb{E}^{\phi}\left[\mathbf{1}_{\left\{T_{n}+Z_{n+1} \leq T\right\}} g\left(X_{T_{n}+Z_{n+1}}^{\phi}\right)+\mathbf{1}_{\left\{T_{n} \leqslant T<T_{n}+Z_{n+1}\right\}} G\left(X_{T}^{\phi}\right)\right]+ \\
& \quad+\mathbb{E}^{\phi}\left[\mathbf{1}_{\left\{T_{n}+Z_{n+1} \leq T\right\}} \mathbb{E}\left[\sum_{h \in I_{n+1}} g\left(X_{h}^{\phi}\right)+G\left(X_{T}^{\phi}\right) \mid Z_{n+1}, X_{T_{n}+Z_{n+1}}^{\phi}\right]\right]= \\
= & \mathbb{E}^{\phi}\left[\mathbf{1}_{\left\{T_{n}+Z_{n+1} \leq T\right\}} g\left(X_{T_{n}+Z_{n+1}}^{\phi}\right)+V^{\phi}\left(T_{n}+Z_{n+1}, X_{T_{n}+Z_{n+1}}^{\phi}\right)+\right. \\
& \left.\quad+\mathbf{1}_{\left\{T_{n} \leqslant T<T_{n}+Z_{n+1}\right\}} G\left(X_{T}^{\phi}\right)\right]= \\
= & \left(T^{\phi} V^{\phi}\right)\left(T_{n}, x\right)
\end{aligned}
$$

Part (ii). Using part (i) and the DP-principle, which states that if a control is optimal on a whole sequence of periods than it has to be optimal on every single period, we obtain:

$$
\begin{aligned}
V^{*} & \left(T_{n}, x\right)=\sup _{\phi \in \Phi} V^{\phi}\left(T_{n}, x\right)=\sup _{\phi \in \Phi} T^{\phi} V^{\phi}\left(T_{n}, x\right)= \\
= & \sup _{\phi \in \Phi} \mathbb{E}_{T_{n}, x}^{\phi}\left[\mathbf{1}_{\left\{T_{n}+Z \leqslant T\right\}} g\left(X_{T_{n}+Z}^{\phi}\right)+V^{\phi}\left(T_{n}+Z, X_{T_{n}+Z}^{\phi}\right)+\mathbf{1}_{\left\{T_{n} \leqslant T<T_{n}+Z\right\}} G\left(X_{T}^{\phi}\right)\right]= \\
= & \sup _{\phi_{T_{n}} \in U} \mathbb{E}_{T_{n}, x}^{\phi_{T_{n}}}\left[\mathbf{1}_{\left\{T_{n}+Z \leqslant T\right\}} g\left(X_{T_{n}+Z}^{\phi_{T_{n}}}\right)+\mathbf{1}_{\left\{T_{n} \leqslant T<T_{n}+Z\right\}} G\left(X_{T}^{\phi_{T_{n}}}\right)+\right. \\
& \left.\quad+\sup _{\phi=\left\{\phi_{T_{i}}\right\}_{i \in\left\{n+1, \ldots, N_{T}\right\}} \in \Phi} V^{\phi}\left(T_{n}+Z, X_{T_{n}+Z}^{\phi_{T_{n}}}\right)\right]= \\
= & \sup _{\phi_{T_{n}} \in U} \mathbb{E}_{T_{n}, x}^{\phi_{T_{n}}}\left[\mathbf{1}_{\left\{T_{n}+Z \leqslant T\right\}} g\left(X_{T_{n}+Z}^{\phi_{T_{n}}}\right)+\mathbf{1}_{\left\{T_{n} \leqslant T<T_{n}+Z\right\}} G\left(X_{T}^{\phi_{T_{n}}}\right)+V^{*}\left(T_{n}, X_{T_{n}+Z}^{\phi_{T_{n}}}\right)\right]= \\
= & \left(T^{*} V^{*}\right)\left(T_{n}, x\right)
\end{aligned}
$$

Corollary 12. $V^{\phi}$ is a fixed point for the map $T^{\phi}$ and $V^{*}$ is a fixed point for the map $T^{*}$. 
Example 13. With the assumptions of Section 2, if we denote the joint probability distribution function of the discrete random variables $(W, Y)$ by

$$
\mu(w, y):=\mathbb{P}[W=w, Y=y]=\mathbb{P}[W=w] \cdot \mathbb{P}[Y=y]
$$

the expanded form of formula (13) with $v(t, x)=V^{\phi}(t, x)$ is:

$$
\begin{aligned}
& \left(T^{\phi} V^{\phi}\right)(t, x)= \\
& \int_{0}^{T-t} \lambda e^{-\lambda \xi}\left(\sum _ { \substack { y \in \{ 0 , \overline { y } \} \\
w \in \{ - d , 0 , u \} } } \mu ( w , y ) \cdot \left[g\left(x+c(b) \xi-b y+\delta\left(e^{w}-1\right)\right)+\right.\right. \\
& \left.\left.\quad+V^{\phi}\left(t+\xi, x+c(b) \xi-b y+\delta\left(e^{w}-1\right)\right)\right]\right) d \xi+ \\
& \quad+\int_{T-t}^{+\infty} \lambda e^{-\lambda \xi} G(x+c(b)(T-t)) d \xi= \\
& =\int_{0}^{T-t} \lambda e^{-\lambda \xi}\left(\sum _ { y , w } \mu ( w , y ) \cdot \left[g\left(x+c(b) \xi-b y+\delta\left(e^{w}-1\right)\right)+\right.\right. \\
& \left.\left.\quad+V^{\phi}\left(t+\xi, x+c(b) \xi-b y+\delta\left(e^{w}-1\right)\right)\right]\right) d \xi+ \\
& \quad+e^{-\lambda(T-t)} G(x+c(b)(T-t))
\end{aligned}
$$

Notice that, even if "a priori" formula (13) requires an integration of $v\left(t+\xi, X_{T_{n+1}}\right)$ for $\xi \in$ $[0, \infty]$, thanks to the definition of $V^{\phi}(t, x)$ in formula (11), we can integrate $V^{\phi}\left(t+\xi, X_{T_{n+1}}\right)$ for $\xi \in[0, T-t]$, having $V^{\phi}\left(t+\xi, X_{T_{n+1}}\right)=0$ when $t+\xi>T$.

\subsection{Contraction property of the operators}

This section is devoted to show that the operators $T^{\phi}$ and $T^{*}$ are contraction mappings on the metric space $\mathcal{B}\left(\mathbb{R}^{+} \times \mathbb{R}\right)$ endowed with the sup-norm

$$
\|v\|_{\infty}=\sup _{(t, x) \in \mathbb{R}^{+} \times \mathbb{R}}|v(t, x)|
$$

We recall that a map $T$ is a contraction if there is a real number $q<1$ such that for all functions $v, v^{\prime} \in \mathcal{B}\left(\mathbb{R}^{+} \times \mathbb{R}\right)$ one has:

$$
\left\|T v-T v^{\prime}\right\|_{\infty} \leqslant q \cdot\left\|v-v^{\prime}\right\|_{\infty}
$$

For brevity we denote $\mathcal{C}_{\mathcal{B}}=\mathcal{C}_{\mathcal{B}}\left(\mathbb{R}^{+} \times \mathbb{R}\right)$ and $\|*\|=\|*\|_{\infty}$.

Assumption 14. The functions $g$ and $G$ satisfy Assumption 6 and are continuous.

Proposition 15. Let $v \in \mathcal{C}_{\mathcal{B}}$. Under Assumption 14 the following hold:

i. for all $\phi \in \Phi$ that are continuous functions of their arguments, namely $\phi=\phi(t, x)$ a continuous function of $(t, x)$, one has $T^{\phi} v \in \mathcal{C}_{\mathcal{B}}$, i.e. $T^{\phi}: \mathcal{C}_{\mathcal{B}} \longrightarrow \mathcal{C}_{\mathcal{B}}$.

ii. the optimal control $\phi^{*}(t, x)=\arg \sup _{\phi_{N_{t}} \in U}\left(T^{\phi} v\right)(t, x)$ exists and is a continuous function of its arguments.

iii. for all $m \in \mathbb{N}$ one has $v_{m}^{*}(t, x) \in \mathcal{C}_{\mathcal{B}}$, where the functions $v_{m}^{*}(t, x)$ are defined in formula (17). 
Proof. Given $t \in[0, T]$ and a sequence $t_{n} \in[0, T]$ such that $\lim _{n \rightarrow \infty} t_{n}=t$, notice first that, thanks to the fact that $Z$ is a continuous random variable, we are allowed to write:

$$
\lim _{n \rightarrow+\infty} \mathbf{1}_{\left\{t_{n}+Z<T\right\}}=\mathbf{1}_{\{t+Z \leqslant T\}}=\mathbf{1}_{\{t+Z<T\}} \quad \text { a.s. }
$$

Part (i). Let $\psi \in \mathbb{R}^{+}$s.t. $\|v\| \leqslant \psi$; then $T^{\phi}$ is bounded:

$$
\begin{aligned}
\left\|\left(T^{\phi} v\right)\right\|= & \left\|\mathbb{E}_{t, x}^{\phi}\left[\mathbf{1}_{\{t+Z<T\}} g\left(X_{t+Z}^{\phi}\right)+v\left(t+Z, X_{t+Z}^{\phi}\right)+\mathbf{1}_{\{t \leqslant T<t+Z\}} G\left(X_{T}^{\phi}\right)\right]\right\| \leqslant \\
\leqslant & \mathbb{E}_{t, x}^{\phi}\left[\left\|\mathbf{1}_{\{t+Z\}<T\}}\right\| \cdot\left\|g\left(X_{t+Z}^{\phi}\right)\right\|+\left\|v\left(t+Z, X_{t+Z}^{\phi}\right)\right\|+\right. \\
& \left.+\left\|\mathbf{1}_{\{t \leqslant T<t+Z\}}\right\| \cdot\left\|G\left(X_{T}^{\phi}\right)\right\|\right] \leqslant \mathcal{G}+\psi+\mathcal{G}
\end{aligned}
$$

To prove the continuity of $T^{\phi} v$, it suffices to show that for each $\left\{\left(t_{n}, x_{n}\right)\right\}_{n \in \mathbb{N}} \subset[0, T] \times \mathbb{R}$ s.t. $\lim _{n \rightarrow \infty}\left(t_{n}, x_{n}\right)=(t, x)$ one has $\lim _{n \rightarrow \infty}\left(T^{\phi} v\right)\left(t_{n}, x_{n}\right)=\left(T^{\phi} v\right)(t, x)$. Notice first that we can exchange the limit with the expected value by the dominated convergence theorem because by assumption $g, G$ and so also $v$ are bounded. Using also formula (20) and the continuity of $c(b)$ we obtain:

$$
\begin{aligned}
& \lim _{n \rightarrow \infty}\left(T^{\phi} v\right)\left(t_{n}, x_{n}\right)= \\
&=\lim _{n \rightarrow \infty}\left(\mathbb{E}_{t_{n}, x_{n}}^{\phi}\left[\mathbf{1}_{\left\{t_{n}+Z<T\right\}} g\left(X_{t_{n}+Z}^{\phi}\right)+v\left(t_{n}+Z, X_{t_{n}+Z}^{\phi}\right)+\mathbf{1}_{\left\{t_{n} \leqslant T<t_{n}+Z\right\}} G\left(X_{T}^{\phi}\right)\right]\right)= \\
&=\lim _{n \rightarrow \infty}\left(\mathbb { E } \left[\mathbf{1}_{\left\{t_{n}+Z<T\right\}} g\left(x_{n}+c\left(b\left(t_{n}, x_{n}\right)\right) Z-b\left(t_{n}, x_{n}\right) Y+\delta\left(t_{n}, x_{n}\right)\left(e^{W}-1\right)\right)\right.\right. \\
& \quad+v\left(t_{n}+Z, x_{n}+c\left(b\left(t_{n}, x_{n}\right)\right) Z-b\left(t_{n}, x_{n}\right) Y+\delta\left(t_{n}, x_{n}\right)\left(e^{W}-1\right)\right) \\
&\left.\quad+\mathbf{1}_{\left\{t_{n} \leqslant T<t_{n}+Z\right\}} G\left(x_{n}+c\left(b\left(t_{n}, x_{n}\right)\right)\left(T-t_{n}\right)\right]\right)= \\
&=\mathbb{E}\left[\mathbf{1}_{\{t+Z<T\}} g\left(x+c(b(t, x)) Z-b(t, x) Y+\delta(t, x)\left(e^{W}-1\right)\right)+\right. \\
& \quad+v\left(t+Z, x+c(b(t, x)) Z-b(t, x) Y+\delta(t, x)\left(e^{W}-1\right)\right) \\
&\left.\quad+\mathbf{1}_{\{t \leqslant T<t+Z\}} G(x+c(b(t, x))(T-t))\right]= \\
&=\mathbb{E}_{t, x}^{\phi}\left[\mathbf{1}_{\{t+Z<T\}} g\left(X_{t+Z}^{\phi}\right)+v\left(t+Z, X_{t+Z}^{\phi}\right)+\mathbf{1}_{\{t \leqslant T<t+Z\}} G\left(X_{T}^{\phi}\right)\right]=\left(T^{\phi} v\right)(t, x)
\end{aligned}
$$

Having assumed that $\phi=\phi(t, x)$ is a continuous function of its arguments and recalling from Remark 2 that $\phi(t, x)=(b(t, x), \delta(t, x))$, the expressions $b\left(t_{n}, x_{n}\right), \delta\left(t_{n}, x_{n}\right)$ used in the above formula are simply the continuous functions $b(t, x), \delta(t, x)$ evaluated at $\left(t_{n}, x_{n}\right)$.

Part (ii). First of all note that $\phi \in U$ and $U=[0,1] \times\left[-C_{1}, C_{2}\right]$ is compact. So, if we show that $\forall(t, x) \in[0, T] \times \mathbb{R}$ the function $\phi \rightarrow\left(T^{\phi} v\right)(t, x)$ is continuous, we can conclude the existence of $\phi^{*}$.

To prove the continuity let $\left\{\phi_{n}\right\}_{n \in \mathbb{N}}=\left\{\left(b_{n}, \delta_{n}\right)\right\}_{n \in \mathbb{N}} \rightarrow(b, \delta)=\phi$; with exactly the same arguments as in part (i), using the continuity of $c(b)$ one gets the result. This implies also that $\phi^{*}$ is a max, not only a sup.

Control's continuity is assured by lemma 3.2 in Onesimo-Hernandez and Runggaldier(1994) (or by the so-called Michael's Theorem, see Michael(1970)).

Part (iii). By induction.

By Assumption 14 the function $G(x)$ is continuous and, using Michael's Theorem, also the optimal control $b^{*}(t, x)$ is a continuous functions. So $v_{0}^{*}(t, x)=G\left(x+c\left(b^{*}(t, x)\right)(T-t)\right)$ is a continuous function.

Now suppose that $v_{m}^{*}(t, x)$ is a continuous function and consider

$$
v_{m+1}^{*}(t, x)=\sup _{\phi \in U}\left(T^{\phi} v_{m}^{*}\right)(t, x)
$$


Thanks to part (ii) of this proof, the optimal control $\phi^{*}$ exists, is a maximum point and is continuous, so also $v_{m+1}^{*}(t, x)$ is a continuous function, being $\left(T^{\phi^{*}} v_{m}^{*}\right)(t, x)$ continuous thanks to part $(i)$.

From the definition of $T^{*}$ in (14) and the proof of the previous proposition, in particular from parts (i) and (ii), we obtain

Corollary 16. Under Assumption 14, for $v \in \mathcal{C}_{\mathcal{B}}$ we have $T^{*} v \in \mathcal{C}_{\mathcal{B}}$, i.e. $T^{*}: \mathcal{C}_{\mathcal{B}} \longrightarrow \mathcal{C}_{\mathcal{B}}$

We are now ready to prove the contraction property of the operators $T^{\phi}$ and $T^{*}$. We have

Proposition 17. For $v, v^{\prime} \in \mathcal{C}_{\mathcal{B}}\left(\mathbb{R}^{+} \times \mathbb{R}\right)$ and under Assumption 14, the maps $T^{\phi}(\forall \phi \subset \Phi)$ and $T^{*}$ are contraction mappings, in particular we have:

i. $\left\|T^{\phi} v-T^{\phi} v^{\prime}\right\| \leqslant\left(1-e^{-\lambda T}\right)\left\|v-v^{\prime}\right\|$

ii. $\left\|T^{*} v-T^{*} v^{\prime}\right\| \leqslant\left(1-e^{-\lambda T}\right)\left\|v-v^{\prime}\right\|$

The mappings $T^{\phi}$ are contracting also in $\mathcal{B}\left(\mathbb{R}^{+} \times \mathbb{R}\right)$ and for this Assumption 6 suffices.

Proof. Let $h(t, x)=v(t, x)-v^{\prime}(t, x)=\left(v-v^{\prime}\right)(t, x)$.

Part (i). For a given $\phi \in \Phi$ it holds:

$$
\begin{aligned}
& \left\|T^{\phi} v-T^{\phi} v^{\prime}\right\|= \\
& =\sup _{(t, x)} \mid \mathbb{E}_{t, x}^{\phi}\left[\mathbf{1}_{\{t+Z<T\}} g\left(X_{t+Z}^{\phi}\right)+v\left(t+Z, X_{t+Z}^{\phi}\right)+\mathbf{1}_{\{t \leqslant T<t+Z\}} G\left(X_{T}^{\phi}\right)\right] \\
& -\mathbb{E}_{t, x}^{\phi}\left[\mathbf{1}_{\{t+Z<T\}} g\left(X_{t+Z}^{\phi}\right)+v^{\prime}\left(t+Z, X_{t+Z}^{\phi}\right)+\mathbf{1}_{\{t \leqslant T<t+Z\}} G\left(X_{T}^{\phi}\right)\right] \\
& \leqslant \sup _{(t, x)} \mathbb{E}_{t, x}^{\phi}\left[\left|h\left(t+Z, X_{t+Z}^{\phi}\right)\right|\right]= \\
& =\sup _{(t, x)} \int_{0}^{T-t} \lambda e^{-\lambda \xi} \sum_{y, w} \mu(w, y)\left|h\left(t+\xi, x+c(b) \xi-b y+\delta\left(e^{w}-1\right)\right)\right| d \xi \leqslant \\
& \leqslant \sup _{(t, x)} \int_{0}^{T-t} \lambda e^{-\lambda \xi}\left(\sum_{y, w} \mu(w, y)\right)\|h\| d \xi=\sup _{t \in[0, T]}\left(1-e^{-\lambda(T-t)}\right)\|h\|= \\
& =\left(1-e^{-\lambda T}\right)\left\|v-v^{\prime}\right\|
\end{aligned}
$$

Since $\left(1-e^{-\lambda T}\right)<1$ we have that $T^{\phi}$ is, under Assumption 14 , a contraction on $\mathcal{C}_{\mathcal{B}}$; under Assumption 6 it is contracting on $\mathcal{B}\left(\mathbb{R}^{+} \times \mathbb{R}\right)$

Part (ii). Given $v, v^{\prime} \in \mathcal{C}_{\mathcal{B}}$, let:

$$
\begin{aligned}
& \phi^{*}(t, x)=\arg \left(\max _{\phi_{T_{N_{t}}} \in U}\left(T^{\phi} v\right)(t, x)\right) \\
& \varphi^{*}(t, x)=\arg \left(\max _{\phi_{T_{N_{t}}} \in U}\left(T^{\phi} v^{\prime}\right)(t, x)\right)
\end{aligned}
$$


where the max exists by Proposition 15ii). Since $\left(T^{\varphi^{*}} v^{\prime}\right) \geqslant\left(T^{\phi^{*}} v^{\prime}\right)$ and $\left(T^{\phi^{*}} v\right) \geqslant\left(T^{\varphi^{*}} v\right)$ it holds:

$$
\begin{aligned}
\left(T^{*} v-T^{*} v^{\prime}\right)(t, x) & =\left(T^{\phi^{*}} v-T^{\varphi^{*}} v^{\prime}\right)(t, x) \leqslant \\
& \leqslant\left(T^{\phi^{*}} v-T^{\phi^{*}} v^{\prime}\right)(t, x) \leqslant \\
& \leqslant\left\|T^{\phi^{*}} v-T^{\phi^{*}} v^{\prime}\right\| \leqslant\left(1-e^{-\lambda T}\right)\left\|v-v^{\prime}\right\| \\
\left(T^{*} v^{\prime}-T^{*} v\right)(t, x) & =\left(T^{\varphi^{*}} v^{\prime}-T^{\phi^{*}} v\right)(t, x) \leqslant \\
& \leqslant\left(T^{\varphi^{*}} v^{\prime}-T^{\varphi^{*}} v\right)(t, x) \leqslant \\
& \leqslant\left\|T^{\varphi^{*}} v^{\prime}-T^{\varphi^{*}} v\right\|\left(1-e^{-\lambda T}\right)\left\|v^{\prime}-v\right\|
\end{aligned}
$$

So $\forall(t, x) \in[0, T] \times \mathbb{R}$ we have $\left|\left(T^{*} v-T^{*} v^{\prime}\right)(t, x)\right| \leqslant\left(1-e^{-\lambda T}\right)\left\|v-v^{\prime}\right\|$ and the conclusion follows.

Since $\mathcal{B}(\cdot)$ and $\mathcal{C}_{\mathcal{B}}(\cdot)$ are complete, separable metric spaces, from the proof of the previous proposition we have

Corollary 18. For all $\phi \in \Phi$, the mappings $T^{\phi}$ have, under Assumption 6 , a unique fixed point in $\mathcal{B}\left(\mathbb{R}^{+} \times \mathbb{R}\right)$ and, see Theorem $11, V^{\phi}(t, x)=\left(T^{\phi} V^{\phi}\right)(t, x)$. If $\phi=\phi(t, x)$ with $\phi(t, x)$ a continuous function of its arguments then, under Assumption 14, the fixed point belongs to $\mathcal{C}_{\mathcal{B}}$. The mapping $T^{*}$ has, always under Assumption 14 and by Proposition 15.ii) (see also (16)), a fixed point in $\mathcal{C}_{\mathcal{B}}$ and, see Theorem $11, V^{*}(t, x)=\left(T^{*} V^{*}\right)(t, x)$.

\subsection{The Value Iteration Algorithm}

In this subsection we let Assumption 14 be in force. We also need some new notations: we recall that a strategy $\phi$ has the form $\phi=\left(\phi_{1}, \ldots \phi_{N_{T}}\right) \in \Phi$; we may also consider the additional component $\phi_{T_{N_{T}}+1}$ that however reduces to $b_{T_{N_{T}+1}}$ (see (8)).

Notation 19. We shall use the following:

- Let $\Phi^{m} \subset \Phi$ the set of admissible strategies with only $m$ components, namely relative to the problem where at most $m$ jump times are considered. Denote its elements by $\phi^{m}$, more precisely:

$$
\phi^{m}=\left(\phi_{1}^{m}, \phi_{2}^{m}, \ldots, \phi_{m}^{m}\right) \in \Phi^{m}
$$

- Given $\phi \in \Phi$, denote by $\phi^{\mid m} \in \Phi^{m}$ its restriction to the first $m$ components, i.e. if $\phi=$ $\left(\phi_{1}, \ldots, \phi_{m}, \phi_{m+1}, \ldots, \phi_{N_{T}}\right)$ then $\phi^{\mid m}=\left(\phi_{1}, \ldots, \phi_{m}\right)$.

- Denote by $\left(T^{*}\right)^{m}=\left(T^{*} \circ \cdots \circ T^{*}\right)$ the operator $T^{*}$ composed m-times. It corresponds to the $m$-th iterate of $T^{*}$, so we can write (see (17)):

$$
v_{m}^{*}(t, x)=\left(\left(T^{*} \circ \cdots \circ T^{*}\right) v_{0}^{*}\right)(t, x)=\left(\left(T^{*}\right)^{m} v_{0}^{*}\right)(t, x)
$$

Definition 20. The optimal strategy obtained from the first $m$ iterations of $T^{*}$ is:

$$
\phi^{*, m}=\arg \max _{\phi^{m} \in \Phi^{m}} v_{m}^{\phi^{m}}
$$

where $v_{m}^{\phi}$ is as defined in formula (16).

Remark 21. $\phi^{m} \in \Phi^{m}$ can always be extended to $\phi \in \Phi$ by adding e.g. zero-valued components. 
The following corollary is, combined with Corollary 18, a summary of the results obtained so far and suggests an iterative algorithm to get an approximate optimum value and an approximate optimal strategy.

\section{Corollary 22. Under Assumption 14 it holds that}

i. for all $\phi \in \Phi$ the value function $V^{\phi}$ is the unique fixed point of $T^{\phi}$ in $\mathcal{B}\left(\mathbb{R}^{+}, \mathbb{R}\right)$; if $\phi=\phi(t, x)$ is a continuous function of $(t, x)$, then $V^{\phi}$ belongs to $\mathcal{C}_{\mathcal{B}}\left(\mathbb{R}^{+}, \mathbb{R}\right)$;

ii. $V^{*}$ is the unique fixed point of $T^{*}$ in $\mathcal{C}_{\mathcal{B}}\left(\mathbb{R}^{+}, \mathbb{R}\right)$;

iii. the strategy $\tilde{\phi}=\arg \max _{\phi \in U} T^{\phi} V^{*}$ is optimal;

iv. $\forall \phi \in \Phi$, given $\varepsilon>0, \exists m_{\varepsilon}>0$ s.t. $\forall m>m_{\varepsilon}$

$$
\left\|V^{\phi}-v_{m}^{\phi^{\mid m}}\right\|<\varepsilon
$$

In particular, it suffices to take:

$$
m_{\varepsilon}>\frac{\log \left(\frac{\varepsilon}{4 \mathcal{G}}\right)-\lambda T}{\log \left(1-e^{-\lambda T}\right)}
$$

v. given $\varepsilon>0, \exists m_{\varepsilon}>0$ s.t. $\forall m>m_{\varepsilon}$

$$
\left\|V^{*}-v_{m}^{\phi^{*}, m}\right\|=\left\|\sup _{\phi \in \Phi} V^{\phi}-\sup _{\phi^{m} \in \Phi^{m}} v_{m}^{\phi^{m}}\right\|<\varepsilon
$$

Proof. Part (i) and Part (ii) follow directly from Corollary 18.

Part (iii): Being

$$
\sup _{\phi \in U}\left(T^{\phi} V^{*}\right)=T^{*} V^{*}=V^{*}
$$

the strategy $\tilde{\phi}=\arg \max _{\phi \in U} T^{\phi} V^{*}$ is optimal (remember that $V^{*}=V^{\phi^{*}}$ where $\phi^{*}$ is the optimal strategy) and it exists by virtue of Proposition 15 .

Part (iv): By the fixed point equation it is obvious that $\left\|V^{\phi}-v_{m}^{\phi \mid m}\right\| \longrightarrow 0$.

The following proof derives formula (23). Recalling that $V^{\phi}$ is the unique fixed point of $T^{\phi}$ (part (i) above) we may write $V^{\phi}=\lim _{n}\left(T^{\phi} \circ \ldots \circ T^{\phi}\right)^{n} v_{0}^{\phi}=\lim _{n} v_{n}^{\phi}$ and also, using telescopic series and the triangular inequality:

$$
\left\|V^{\phi}-v_{m}^{\phi}\right\|=\left\|\sum_{k=m}^{+\infty}\left(v_{k+1}^{\phi}-v_{k}^{\phi}\right)\right\| \leqslant \sum_{k=m}^{+\infty}\left\|v_{k+1}^{\phi}-v_{k}^{\phi}\right\|
$$

Thanks to Proposition 17 we can easily estimate the norm $\left\|v_{k+1}^{\phi}-v_{k}^{\phi}\right\|$ :

$$
\begin{aligned}
\left\|v_{k+1}^{\phi}-v_{k}^{\phi}\right\| & =\left\|\left(T^{\phi} \circ \cdots \circ T^{\phi}\right)^{k}\left(v_{1}^{\phi}-v_{0}^{\phi}\right)\right\|= \\
& =\left\|\left(T^{\phi} \circ \cdots \circ T^{\phi}\right)^{k}\left(T^{\phi} v_{0}^{\phi}-v_{0}^{\phi}\right)\right\| \leqslant \\
& \leqslant\left(1-e^{-\lambda T}\right)^{k}\left\|T^{\phi} v_{0}^{\phi}-v_{0}^{\phi}\right\| \leqslant \\
& \leqslant\left(1-e^{-\lambda T}\right)^{k}\left(\left\|T^{\phi} v_{0}^{\phi}\right\|+\left\|v_{0}^{\phi}\right\|\right) \leqslant \\
& \leqslant\left(1-e^{-\lambda T}\right)^{k}(3 \mathcal{G}+\mathcal{G})=4 \mathcal{G}\left(1-e^{-\lambda T}\right)^{k}
\end{aligned}
$$


where in the last inequality we have used the Assumption 6, implied by Assumption 14, and the explicit form of $T^{\phi}$ (formula (13)) and of $v_{0}^{\phi}$ (formula (16)). Using the sum of a geometric series we then obtain:

$$
\begin{aligned}
\left\|V^{\phi}-v_{m}^{\phi}\right\| & \leqslant 4 \mathcal{G} \sum_{k=m}^{+\infty}\left(1-e^{-\lambda T}\right)^{k}=4 \mathcal{G} \sum_{k=1}^{+\infty}\left(1-e^{-\lambda T}\right)^{k+m}= \\
& =4 \mathcal{G}\left(1-e^{-\lambda T}\right)^{m} \sum_{k=1}^{+\infty}\left(1-e^{-\lambda T}\right)^{k}=\frac{4 \mathcal{G}\left(1-e^{-\lambda T}\right)^{m}}{1-\left(1-e^{-\lambda T}\right)}= \\
& =4 \mathcal{G}\left(1-e^{-\lambda T}\right)^{m} e^{\lambda T}
\end{aligned}
$$

Recalling that $\log \left(1-e^{-\lambda T}\right)<0$, formula (23) is the solution of:

$$
4 \mathcal{G}\left(1-e^{-\lambda T}\right)^{m_{\varepsilon}} e^{\lambda T}<\varepsilon
$$

namely

$$
m_{\varepsilon}>\frac{\log \left(\frac{\varepsilon}{4 \mathcal{G}}\right)-\lambda T}{\log \left(1-e^{-\lambda T}\right)}
$$

Part (v). Obvious by the fixed point equation being, see (21), $v_{m}^{\phi^{*, m}}=v_{m}^{*}$.

Remark 23. Part (iii) provides a possible way to find the optimal strategy, but it requires knowledge of the fixed point $V^{*}$, that in general we do not have. We shall have it however in the particular case of Section 4 where we shall then use (25). In the general case $V^{*}$ can only be computed as the limit of $\left(T^{*}\right)^{m} v_{0}^{*}$ where, in practice, the iterations have to be stopped at a finite value of $m$. We shall now estimate the error committed by stopping the iteration algorithm.

The approximation result is

Theorem 24. Under Assumption 14, let $m_{\varepsilon}$ be such that $\forall m>m_{\varepsilon}$ we have that $\left\|V^{*}-v_{m}^{*}\right\|<\varepsilon$ where $v_{m}^{*}$ is as defined recursively in (17). For a given $m>m_{\varepsilon}$ let $\phi^{*, m}$ be the strategy obtained from the first $m$ iterations of $T^{*}$ :

$$
\phi^{*, m}=\arg \max _{\phi^{m} \in \Phi^{m}} v_{m}^{\phi^{m}}
$$

( $\phi^{*, m}$ exists under Assumption 14 and the compactness of $U$ by Proposition 15) and let $\hat{\phi} \in \Phi$ be any extension of $\phi^{*, m}$, i.e.:

$$
\phi^{*, m}=\hat{\phi}^{\mid m}
$$

Then it holds that

$$
\left\|V^{*}-V^{\hat{\phi}}\right\| \leqslant 2 \varepsilon
$$

Proof. We use formula (24) and formula (22) to obtain:

$$
\left\|V^{*}-V^{\hat{\phi}}\right\|=\left\|V^{*}-v_{m}^{\phi^{*, m}}+v_{m}^{\phi^{*, m}}-V^{\hat{\phi}}\right\| \leqslant\left\|V^{*}-v_{m}^{\phi^{*, m}}\right\|+\left\|v_{m}^{\phi^{*, m}}-V^{\hat{\phi}}\right\| \leqslant \varepsilon+\varepsilon=2 \varepsilon
$$

\subsection{The (approximate) strategy}

Given the state $x=X_{0} \in \mathbb{R}$ at time $T_{0}=0$ and given an $m \in \mathbb{N}$, the strategy

$$
\phi^{*, m}=\left(\phi_{1}^{*, m}(0, x), \cdots, \phi_{m}^{*, m}\left(T_{m-1}, X_{T_{m-1}}^{\left(\phi_{1}^{m}, \phi_{2}^{m}, \cdots, \phi_{m-1}^{m}\right)}\right)\right) \in \Phi^{m}
$$

results from iterating $m$ times the operator $T^{*}$ starting from

$$
v_{0}^{*}\left(T_{n}, x\right)=\mathbf{1}_{\left\{T_{n} \leqslant T\right\}} \sup _{b \in[0,1]} G\left(x+c(b)\left(T-T_{n}\right)\right)
$$

So: 
- $v_{0}^{*}$ represents the maximum expected cumulative utility when there are no more jumps before $T$, i.e. we are sitting at time $T_{m-1}$; at that time the capital will be $X_{T_{m-1}}^{\left(\phi_{1}^{*, m}, \phi_{2}^{*, m}, \cdots, \phi_{m-1}^{*, m}\right)}=$ $X_{T_{m-1}}$ and so $\phi_{T_{m}}^{*, m}\left(T_{m-1}, X_{T_{m-1}}\right)=\arg \max _{\phi} v_{0}^{\phi}\left(T_{m-1}, X_{T_{m-1}}\right)$.

- $\phi_{1}^{*, m}\left(T_{0}, X_{T_{0}}\right)$ results from the last iteration of $T^{*}$ with $T_{0}=0, X_{0}=x$, namely from computing

$$
\begin{aligned}
\sup _{(b, \delta)}\left\{\int_{0}^{T} \lambda e^{-\lambda \xi}\right. & \left(\sum _ { y , w } \mu ( w , y ) \cdot \left[g\left(x+c(b) \xi-b y+\delta\left(e^{w}-1\right)\right)+\right.\right. \\
& \left.\left.\left.+v_{m-1}^{*}\left(\xi, x+c(b) \xi-b y+\delta\left(e^{w}-1\right)\right)\right]\right) d \xi+e^{-\lambda T} G(x+c(b) T)\right\}
\end{aligned}
$$

- in general, for $i \in\{1, m\}, \phi_{i}^{*, m}\left(T_{i-1}, X_{T_{i-1}}\right)$ results from the $(m-i)$-th iteration of $T^{*}$ and it is given as

$$
\arg \max _{\phi \in U}\left(T^{\phi} v_{m-i}^{*}\right)\left(T_{i-1}, X_{T_{i-1}}\right)
$$

- for $i \in\left\{m, \ldots, N_{T}\right\}$ the strategy is completed, e.g. by adding zero-valued components.

\section{Computing the solution via value iteration}

This section is divided into two subsections.

- Subsection 4.1 is devoted to the study of the problem when the utility functions $g$ and $G$ are of the exponential type and for which an analytic solution can be obtained.

- In subsection 4.2 we mention a numerical algorithm which performs the iterates of $T^{*}$ in the case of two generic utility functions $g$ and $G$.

\subsection{Semianalytic solutions for exponential utility functions}

Let (exponential utility functions)

$$
g(x)=1-\gamma e^{-\beta x} \quad, \quad G(x)=1-\mu e^{-\beta x}
$$

for some constants $\gamma, \mu, \beta \in \mathbb{R}^{+}, \beta \neq 0$. We notice that if the size of the surplus/risk process is negative or small then the associated utility is negative or small and so the maximization of the expected total utility can be viewed as an "implicit" minimization of the ruin probability. The above functions, independent of $\phi$ and $t$, satisfy the Assumptions 6 and 14 for $x \in \mathbb{R}$, so $V^{\phi}\left(T_{n}, x\right)$ is increasing in $x$, bounded and continuous.

\subsubsection{Fixed Point Characterization}

Definition 25. Let

$$
\mathcal{V}=\left\{v: \mathbb{R}^{+} \times \mathbb{R} \longrightarrow \mathbb{R} \quad \text { s.t. } \quad v(t, x)=\mathbf{1}_{\{t \leqslant T\}}\left(M(t)-e^{-\beta x} \nu(t)\right) ; \quad \nu(t)>0, \beta>0\right\}
$$

We now prove that, given $g$ and $G$ as in (26), the set $\mathcal{V}$ is closed under the action of $T^{\phi}$ and $T^{*}$. This leads to what in Schäl(2004) is called "structure assumption" for the value iteration. First we consider $T^{\phi}$ in the following 
Theorem 26. For a fixed $\phi=(b, \delta) \in \Phi$, given $g, G$ as in formula (26), the set $\mathcal{V}$ is closed under $T^{\phi}$, i.e. if

$$
v(t, x)=\mathbf{1}_{\{t \leqslant T\}}\left(M(t)-e^{-\beta x} \nu(t)\right)
$$

then there exists $\tilde{M}(t)$ and $\tilde{\nu}(t)>0$ s.t.

$$
\left(T^{\phi} v\right)(t, x)=\mathbf{1}_{\{t \leqslant T\}}\left(\tilde{M}(t)-e^{-\beta x} \tilde{\nu}(t)\right)
$$

Proof. Let $v \in \mathcal{V}$ for some given $M(t), \nu(t)$ and define: $\chi=\chi_{Z}^{\phi}=c(b) Z-b Y+\delta\left(e^{W}-1\right)$.

$$
\begin{aligned}
& \left(T^{\phi} v\right)(t, x)= \\
& =\mathbf{1}_{\{t \leqslant T\}} \mathbb{E}_{t, x}^{\phi}\left[\mathbf{1}_{\{t+Z \leqslant T\}} g\left(X_{t+Z}^{\phi}\right)+\mathbf{1}_{\{t+Z \leqslant T\}}\left(M(t+Z)-e^{-\beta X_{t+Z}^{\phi} \nu}(t+Z)\right)+\right. \\
& \left.+\mathbf{1}_{\{t \leqslant T\}} \mathbf{1}_{\{t+Z>T\}} G\left(X_{T}^{\phi}\right)\right]= \\
& =\mathbf{1}_{\{t \leqslant T\}}\left\{\mathbb { E } _ { t , x } ^ { \phi } \left[\mathbf{1}_{\{t+Z \leqslant T\}}\left(1-\gamma e^{-\beta\left(x+c(b) Z-b Y+\delta\left(e^{W}-1\right)\right)}\right)+\right.\right. \\
& +\mathbf{1}_{\{t+Z \leqslant T\}}\left(M(t+Z)-e^{-\beta\left(x+c(b) Z-b Y+\delta\left(e^{W}-1\right)\right)} \nu(t+Z)\right)+ \\
& \left.\left.+\mathbf{1}_{\{t+Z>T\}}\left(1-\mu e^{-\beta(x+c(b)(T-t))}\right)\right]\right\}= \\
& =\mathbf{1}_{\{t \leqslant T\}}\left\{\mathbb { E } _ { t , x } ^ { \phi } \left[\mathbf{1}_{\{t+Z \leqslant T\}}\left(1-\gamma e^{-\beta x-\beta \chi}\right)+\right.\right. \\
& \left.\left.+\mathbf{1}_{\{t+Z \leqslant T\}}\left(M(t+Z)-e^{-\beta x-\beta \chi} \nu(t+Z)\right)+\mathbf{1}_{\{t+Z>T\}}\left(1-\mu e^{-\beta(x+c(b)(T-t))}\right)\right]\right\}= \\
& =\mathbf{1}_{\{t \leqslant T\}}\left\{\mathbb{E}_{t, x}^{\phi}\left[\mathbf{1}_{\{t+Z \leqslant T\}}+\mathbf{1}_{\{t+Z \leqslant T\}} M(t+Z)+\mathbf{1}_{\{t+Z>T\}}\right]+\right. \\
& \left.-\mathbb{E}_{t, x}^{\phi}\left[\mathbf{1}_{\{t+Z \leqslant T\}} \gamma e^{-\beta x-\beta \chi}+\mathbf{1}_{\{t+Z \leqslant T\}} e^{-\beta x-\beta \chi} \nu(t+Z)+\mathbf{1}_{\{t+Z>T\}} \mu e^{-\beta(x+c(b)(T-t))}\right]\right\}= \\
& =\mathbf{1}_{\{t \leqslant T\}}\left\{\mathbb{E}_{t, x}^{\phi}\left[1+\mathbf{1}_{\{t+Z \leqslant T\}} M(t+Z)\right]+\right. \\
& \left.-e^{-\beta x} \mathbb{E}_{t, x}^{\phi}\left[\mathbf{1}_{\{t+Z \leqslant T\}} e^{-\beta \chi}(\gamma+\nu(t+Z))+\mathbf{1}_{\{t+Z>T\}} \mu e^{-\beta c(b)(T-t)}\right]\right\}= \\
& =\mathbf{1}_{\{t \leqslant T\}}\left\{\tilde{M}(t)-e^{-\beta x} \tilde{\nu}(t)\right\} \in \mathcal{V}
\end{aligned}
$$

where:

$$
\begin{aligned}
\tilde{M}(t) & =1+\mathbb{E}_{t, x}^{\phi}\left[\mathbf{1}_{\{t+Z \leqslant T\}} M(t+Z)\right]=1+\mathbb{E}\left[\mathbf{1}_{\{t+Z \leqslant T\}} M(t+Z)\right] \\
\tilde{\nu}(t) & =\mathbb{E}_{t, x}^{\phi}\left[\mathbf{1}_{\{t+Z \leqslant T\}} e^{-\beta \chi}(\gamma+\nu(t+Z))+\mathbf{1}_{\{t+Z>T\}} \mu e^{-\beta c(b)(T-t)}\right]>0
\end{aligned}
$$

Notice that, although we use the notation $\mathbb{E}_{t, x}^{\phi}$, the expectation is over the joint distribution of $(Z, Y, W)$ so that the functions on the left hand side of (28) and (29) do not depend on $x$ but only on $t$ and, for $\tilde{\nu}$, also on the chosen $\phi=(b, \delta)$. In what follows we shall therefore write $\nu(t ; b, \delta)$ and $\tilde{\nu}(t ; b, \delta)$ instead of $\nu(t)$ and $\tilde{\nu}(t)$ respectively.

For a fixed $\phi=(b, \delta) \in \Phi$ we have the following sequence of equalities for equation (29), 
justified by the independence between $Z, Y$ and $W$ :

$$
\begin{aligned}
& \tilde{\nu}(t ; b, \delta)=\mathbb{E}_{t, x}^{\phi}\left[\mathbf{1}_{\{t+Z \leqslant T\}} e^{-\beta \chi}(\gamma+\nu(t+Z ; b, \delta))+\mathbf{1}_{\{t+Z>T\}} \mu e^{-\beta c(b)(T-t)}\right] \\
& =\mathbb{E}^{\phi}\left[\mathbf{1}_{\{t+Z \leqslant T\}} e^{-\beta\left(c(b) Z-b Y+\delta\left(e^{W}-1\right)\right)}(\gamma+\nu(t+Z ; b, \delta))\right]+\mu e^{-(\lambda+\beta c(b))(T-t)} \\
& =\mathbb{E}^{\phi}\left[e^{-\beta\left(\delta\left(e^{W}-1\right)-b Y\right)}\right] \mathbb{E}^{\phi}\left[\mathbf{1}_{\{t+Z \leqslant T\}} e^{-\beta c(b) Z}(\gamma+\nu(t+Z ; b, \delta))\right]+\mu e^{-(\lambda+\beta c(b))(T-t)}
\end{aligned}
$$

Definition 27. Inspired by formulas (28), (29) and (30), given $M(t)$ and $\nu(t ; b, \delta)$, define:

$$
\begin{gathered}
\tilde{M}(t)=1+\mathbb{E}\left[\mathbf{1}_{\{t+Z \leqslant T\}} M(t+Z)\right] \\
\tilde{\nu}(t ; b, \delta)=\mathbb{E}^{\phi}\left[e^{-\beta\left(\delta\left(e^{W}-1\right)-b Y\right)}\right] \mathbb{E}^{\phi}\left[\mathbf{1}_{\{t+Z \leqslant T\}} e^{-\beta c(b) Z}(\gamma+\nu(t+Z ; b, \delta))\right]+ \\
+\mu e^{-(\lambda+\beta c(b))(T-t)}
\end{gathered}
$$

and let $b^{*}$ and $\delta^{*}$ be s.t. $\forall b \in[0,1], \forall \delta \in\left[-C_{1}, C_{2}\right]$

$$
\nu\left(t ; b^{*}, \delta^{*}\right) \leqslant \nu(t ; b, \delta)
$$

i.e.

$$
\left(b^{*}, \delta^{*}\right)=\arg \inf _{(b, \delta)} \nu(t ; b, \delta)
$$

When we need to be more explicit we will write $\tilde{M}_{M}(t)$ and $\tilde{\nu}_{\nu}(t ; b, \delta)$ to point out that $\tilde{M}(t)$ and $\tilde{\nu}(t ; b, \delta)$ in (31) and (32) depend also on the choice of $M(t$ and $\nu(t ; b, \delta)$.

Remark 28. It is remarkable that formula (33) shows that the optimal controls $b^{*}$ and $\delta^{*}$ depend only on time: they are independent on the value $x$ of the reserve.

We get now the result corresponding to Theorem 26 also for $T^{*}$

Corollary 29. $\mathcal{V}$ is closed also under $T^{*}$, namely if $v \in \mathcal{V}$, i.e.

$$
v(t, x)=\mathbf{1}_{\{t \leqslant T\}}\left(M(t)-e^{-\beta x} \nu(t ; b, \delta)\right)
$$

then

$$
\left(T^{*} v\right)(t, x)=\mathbf{1}_{\{t \leqslant T\}}\left[\tilde{M}(t)-e^{-\beta x} \tilde{\nu}\left(t ; b^{*}, \delta^{*}\right)\right]
$$

where $\tilde{M}(t)$ and $\tilde{\nu}\left(t ; b^{*}, \delta^{*}\right)$ are defined by formulas (31) and (32) respectively.

Proof. Theorem 26 leads to:

$$
\begin{aligned}
& \left(T^{*} v\right)(t, x)= \\
= & \sup _{(b, \delta) \in U} \mathbf{1}_{\{t \leqslant T\}}\left\{\tilde{M}(t)-e^{-\beta x} \tilde{\nu}(t ; b, \delta)\right\}=\mathbf{1}_{\{t \leqslant T\}}\left\{\tilde{M}(t)-e^{-\beta x} \inf _{(b, \delta) \in \Phi} \tilde{\nu}(t ; b, \delta)\right\}= \\
= & \mathbf{1}_{\{t \leqslant T\}}\left\{\tilde{M}(t)-e^{-\beta x} \tilde{\nu}\left(t ; b^{*}, \delta^{*}\right)\right\} \in \mathcal{V}
\end{aligned}
$$

From the the fixed point equation (see Corollary 18 and formula (25)) as well as Corollary 29 we have 
Proposition 30. Let $V^{*}(t, x)$ be of the form

$$
V^{*}(t, x)=\mathbf{1}_{\{t \leqslant T\}}\left(M^{*}(t)-e^{-\beta x} \nu^{*}\left(t ; b^{*}, \delta^{*}\right)\right)
$$

for some functions $M^{*}(t)$ and $\nu^{*}(t ; b, \delta)$ satisfying, for given $(b, \delta)=\phi \in U$, the following integral equations:

$$
\begin{gathered}
M^{*}(t)=1+\mathbb{E}\left[\mathbf{1}_{\{t+Z \leqslant T\}} M^{*}(t+Z)\right]=1+\lambda \int_{0}^{T-t} M^{*}(t+\xi) e^{-\lambda \xi} d \xi \\
\nu^{*}(t ; b, \delta)=\mathbb{E}^{\phi}\left[e^{-\beta\left(\delta\left(e^{W}-1\right)-b Y\right)}\right] \mathbb{E}^{\phi}\left[\mathbf{1}_{\{t+Z \leqslant T\}} e^{-\beta c(b) Z}\left(\gamma+\nu^{*}(t+Z ; b, \delta)\right)\right]+ \\
\quad+\mu e^{-(\lambda+\beta c(b))(T-t)}= \\
=\left(\sum_{w, y} e^{-\beta\left(\delta\left(e^{w}-1\right)-b y\right)} \mathbb{P}[W=w, Y=y]\right) \int_{0}^{T-t} \lambda\left(\gamma+\nu^{*}(t+\xi ; b, \delta)\right) e^{-(\beta c(b)+\lambda) \xi} d \xi+ \\
+\mu e^{(\beta c(b)+\lambda)(t-T)}
\end{gathered}
$$

and let $\phi^{*}=\left(b^{*}, \delta^{*}\right)=\arg \inf _{b, \delta} \nu^{*}(t ; b, \delta) \in \Phi$ be as in formula (33).

Then $V^{*}(t, x) \in \mathcal{V}$ is the unique fixed point of $T^{*}$ and $\phi^{*}$ is the optimal strategy.

Proof. By Corollary 29 and formulas (31) and (32) applied with $M=M^{*}$ and $\nu=\nu^{*}$ one obtains the result in a straightforward manner being:

$$
\begin{aligned}
& \left(T^{*} V^{*}\right)(t, x)=\mathbf{1}_{\{t \leqslant T\}}\left\{\tilde{M}_{M^{*}}(t)-e^{-\beta x} \tilde{\nu}_{\nu^{*}}\left(t ; b^{*}, \delta^{*}\right)\right\} \\
& =\mathbf{1}_{\{t \leqslant T\}}\left\{1+\mathbb{E}\left[\mathbf{1}_{\{t+Z \leqslant T\}} M^{*}(t+Z)\right]\right. \\
& \quad-\inf _{\phi=(b, \delta) \in \Phi} e^{-\beta x}\left(\mathbb{E}^{\phi}\left[e^{-\beta\left(\delta\left(e^{W}-1\right)-b Y\right)}\right] \mathbb{E}^{\phi}\left[\mathbf{1}_{\{t+Z \leqslant T\}} e^{-\beta c(b) Z}\left(\gamma+\nu^{*}(t+Z ; b, \delta)\right)\right]+\right. \\
& \left.\left.\quad+\mu e^{-(\lambda+\beta c(b))(T-t)}\right)\right\}= \\
& =\mathbf{1}_{\{t \leqslant T\}}\left(M^{*}(t)-e^{-\beta x} \nu^{*}\left(t ; b^{*}, \delta^{*}\right)\right)=V^{*}(t, x)
\end{aligned}
$$

\subsubsection{Explicit (semianalytic) solution for the case of exponential utilities}

The crucial integral equations (34) and (35) are two special cases of Volterra integral equations for which an explicit (analytical) solution exists. In fact, both (34) and (35) are of the type of the general Volterra equation

$$
f(t)=g(t)+A \int_{a}^{t} e^{\lambda(t-y)} f(y) d y
$$

that admits (see Polyanin and Manzhirov(2008)) the explicit analytic solution

$$
f(t)=g(t)+A \int_{a}^{t} e^{(\lambda+A)(t-y)} g(y) d y
$$


and this leads in the case of (34) to

$$
M(t)=1+\lambda(T-t)
$$

while for (35) we obtain (see Edoli(2009))

$$
\nu(t, b, \delta)=\frac{\lambda \mathcal{E}(b, \delta) \gamma}{\Lambda(b)-\lambda \mathcal{E}(b, \delta)}+e^{(\Lambda(b)-\lambda \mathcal{E}(b, \delta))(t-T)}\left(\mu-\frac{\lambda \mathcal{E}(b, \delta) \gamma}{\Lambda(b)-\lambda \mathcal{E}(b, \delta)}\right)
$$

having put

$$
\begin{aligned}
\mathcal{E}(b, \delta) & =\mathbb{E}^{\phi}\left[e^{-\beta\left(\delta\left(e^{W}-1\right)-b Y\right)}\right]=\sum_{y, w} e^{-\beta\left(\delta\left(e^{w}-1\right)-b y\right)} \mathbb{P}[W=w] \mathbb{P}[Y=y] \\
\Lambda(b) & =\beta c(b)+\lambda
\end{aligned}
$$

To obtain the optimal values $b^{*}, \delta^{*}$ of the controls we have to minimize $\nu(t ; b, \delta)$ with respect to $(b, \delta)$. The analytic approach to perform the minimization leads to computing the derivatives of $\nu(t ; b, \delta)$ with respect to $b$ and $\delta$ that, letting for a fixed $t$,

$$
\begin{aligned}
\mathcal{E}_{\delta} & =\partial_{\delta} \mathcal{E}=\sum_{y, w} \beta\left(1-e^{w}\right) e^{-\beta\left(\delta\left(e^{w}-1\right)-b y\right)} \mathbb{P}[W=w] \mathbb{P}[Y=y] \\
\mathcal{E}_{b}=\partial_{b} \mathcal{E} & =\sum_{y, w} \beta y e^{-\beta\left(\delta\left(e^{w}-1\right)-b y\right)} \mathbb{P}[W=w] \mathbb{P}[Y=y] \\
\Lambda_{b} & =\partial_{b} \Lambda=\beta c^{\prime}(b)=\beta(1+\theta) \frac{\bar{p} \cdot \bar{y}}{\mathbb{E}[\Xi]} \\
\Lambda_{\delta} & =\partial_{\delta} \Lambda=0
\end{aligned}
$$

are given by

$$
\begin{aligned}
\partial_{b} \nu(t)=\lambda & \gamma \frac{\mathcal{E}_{b} \Lambda-\mathcal{E} \Lambda_{b}}{(\Lambda-\lambda \mathcal{E})^{2}}+ \\
& +e^{(\Lambda-\lambda \mathcal{E})(t-T)}\left[\left(\Lambda_{b}-\lambda \mathcal{E}_{b}\right)(t-T)\left(\mu-\frac{\lambda \mathcal{E} \gamma}{\Lambda-\lambda \mathcal{E}}\right)-\lambda \gamma \frac{\mathcal{E}_{b} \Lambda-\mathcal{E} \Lambda_{b}}{(\Lambda-\lambda \mathcal{E})^{2}}\right] \\
\partial_{\delta} \nu(t)=\lambda & \frac{\mathcal{E}_{\delta} \Lambda}{(\Lambda-\lambda \mathcal{E})^{2}}+ \\
& +e^{(\Lambda-\lambda \mathcal{E})(t-T)}\left[\left(-\lambda \mathcal{E}_{\delta}\right)(t-T)\left(\mu-\frac{\lambda \mathcal{E} \gamma}{\Lambda-\lambda \mathcal{E}}\right)-\lambda \gamma \frac{\mathcal{E}_{\delta} \Lambda}{(\Lambda-\lambda \mathcal{E})^{2}}\right]
\end{aligned}
$$

From here one sees that already the first order conditions are quite involved and so we opt rather for a feasible numerical approach that consists in implementing in Matlab the minimization of $\nu(t ; b, \delta)$. This is the reason why we called the approach "semianalytic". We have nevertheless worked out the analytical derivatives since they have been used in order to speed up and increase the accuracy of the algorithm (details are in Edoli (2009)).

We present now some numerical results, where we keep the less influential parameter values fixed, more precisely

$$
T=1, \theta=.5, \beta=.0007, \gamma=.001, \mu=.7, C_{1}=-5, C_{2}=10, \lambda=1000
$$

and focus our attention on the impact of the following parameters: 
i. The size $\bar{y}$ of the claim (for simplicity we had chosen only one nonzero value for the claim size, i.e. a "representative value") and its probability $\bar{p}=\mathbb{P}\left[Y_{T_{i}}=\bar{y}\right]$.

ii. The parameters that drive the market behavior, namely those that characterize the distribution of $W_{T_{i}}$. They are concerned with the fact that we had assumed $W_{T_{i}} \in\{-d, 0, u\}$ with probabilities $p_{1}, p_{2}, p_{3}=1-p_{1}-p_{2}$ respectively.

The numerical results reported below correspond to the intuition that large and frequent claims ( $\bar{y}$ and $\bar{p}$ large) lead to larger levels of reinsurance, while a favorable market situation ( $u$ and $p_{3}$ relatively large) leads to a higher level of investment.

First we consider the Behavior of $\nu(t ; b, \delta)$. We note that for a fixed $t$ it is possible to plot the surface $\nu(t ; b, \delta)$ on the grid $[0,1] \times\left[C_{1}, C_{2}\right]$ : a graphical inspection of the surface can give us an idea of the behavior of the function that we are trying to minimize and also suggests that changes in the values of the parameters in (42) do not lead to significant changes in the shape of $\nu(t ; b, \delta)$, but may occasionally lead to numerical instability (for example a large value of $\beta$ leads to many NAN, i.e. undetermined ratios).

Figure 1(a) shows the shapes of the surface $\nu(0.9, b, \delta)$ for various values of $\bar{p}$ from 0.01 to 0.2: it is clear that the minimum is attained at $\left(b^{*}, \delta^{*}\right)=(0,10)$. Notice that for small values of $\bar{p}$ the surface is quite flat, while for large values of $\bar{p}$ the surface is increasing in $b$, suggesting that when the claims are frequent the optimal choice is a complete reinsurance, i.e. $b^{*}=0$. The optimal control $\delta^{*}=10$ is due to the favorable situation of the market, having set $p_{1}=0.3$, $p_{2}=0, p_{3}=0.7$. Figure $1(\mathrm{~b})$ shows the same experiment but with an unfavorable market situation, obtained with $p_{1}=0.7, p_{2}=0, p_{3}=0.3$ : in this case the optimal control becomes $\left(b^{*}, \delta^{*}\right)=(0,-5)$.

Next we consider the Optimal controls. An interesting question, suggested by the results on the behavior of $\nu(t ; b, \delta)$, is to investigate the dependence on time of the optimal controls $b^{*}$ and $\delta^{*}$ (recall from Remark 28 that for exponential utilities they depend at most on the time $t$, but not on the level $x$ of the reserve).

In the specific case considered in the numerical calculations the probabilities $p_{1}, p_{2}, p_{3}$ (the main ingredients for the distribution of $W$ ) are supposed to be given and constant over time. It follows that a favorable or unfavorable market situation will remain such throughout and so it does not come at a surprise if the optimal level of investment in the Figures 3(a) and 3(b) remains constant over time. From the numerical calculations it also turned out that as soon as $p_{1}<0.5$ (with $p_{2}=0$ ), namely the market situation is favorable, then the optimal investment corresponds to the highest level. Otherwise, for $p_{1}>0.5$, it corresponds to the lowest level.

For a more general investigation an analytical study of the sign of the derivatives does not seem to be a good way to proceed; however, numerical calculations suggest that the sign of $\partial_{b} \nu(t ; b, \delta)$ and $\partial_{\delta} \nu(t ; b, \delta)$ does not depend on time: when $t=T$ it is immediately seen from (40) and (41) that $\partial_{b} \nu(t ; b, \delta)=\partial_{\delta} \nu(t ; b, \delta)=0$; when $t \in[0, T)$ the sign is always positive or negative, depending on the values of the parameters.

Figures 2(a) and 2(b) show the surfaces of $\partial_{b} \nu(t ; b, \delta)$ and $\partial_{\delta} \nu(t ; b, \delta)$ for some values of $t$; here $\bar{p}=0.01, p_{1}=0.7, p_{2}=0, p_{3}=0.3$ : with this choice of parameters the optimal controls, obtained from a numerical minimization of $\nu(t ; b, \delta)$ on a grid of 100 points in $[0,1]$, are plotted in figures 3(a) and 3(b). Notice also that (37) implies that at $t=T$ one has $\nu(T ; b, \delta) \equiv \mu$ and so for $t=T$ every choice of $b$ and $\delta$ is optimal; in the iterative optimization algorithm the optimal controls are thus simply given by their starting values. 


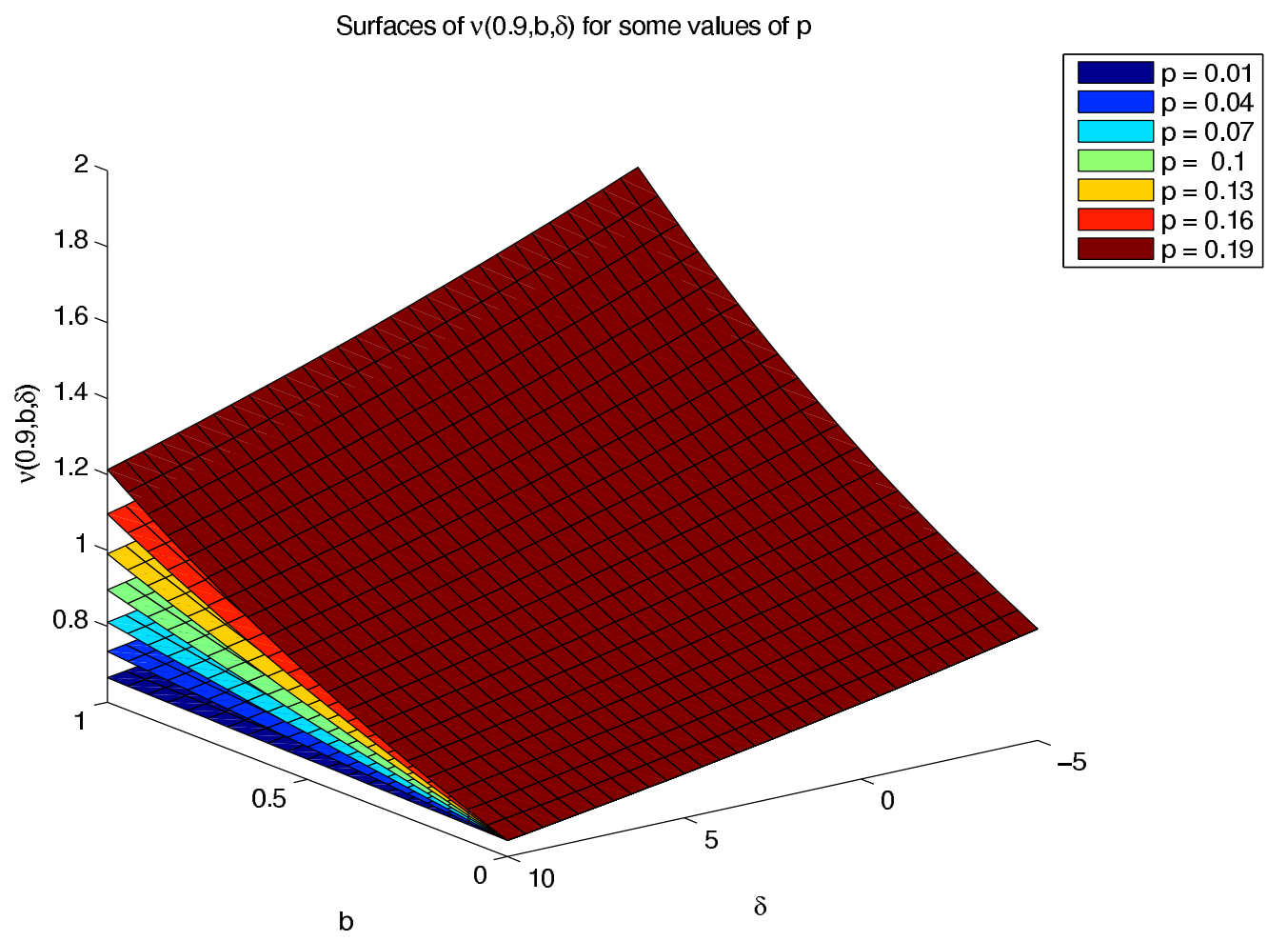

(a) Surface of $\nu(0.9, b, \delta)$ with $p_{1}=0.3, p_{2}=0, p_{3}=0.7, d=u=0.5$

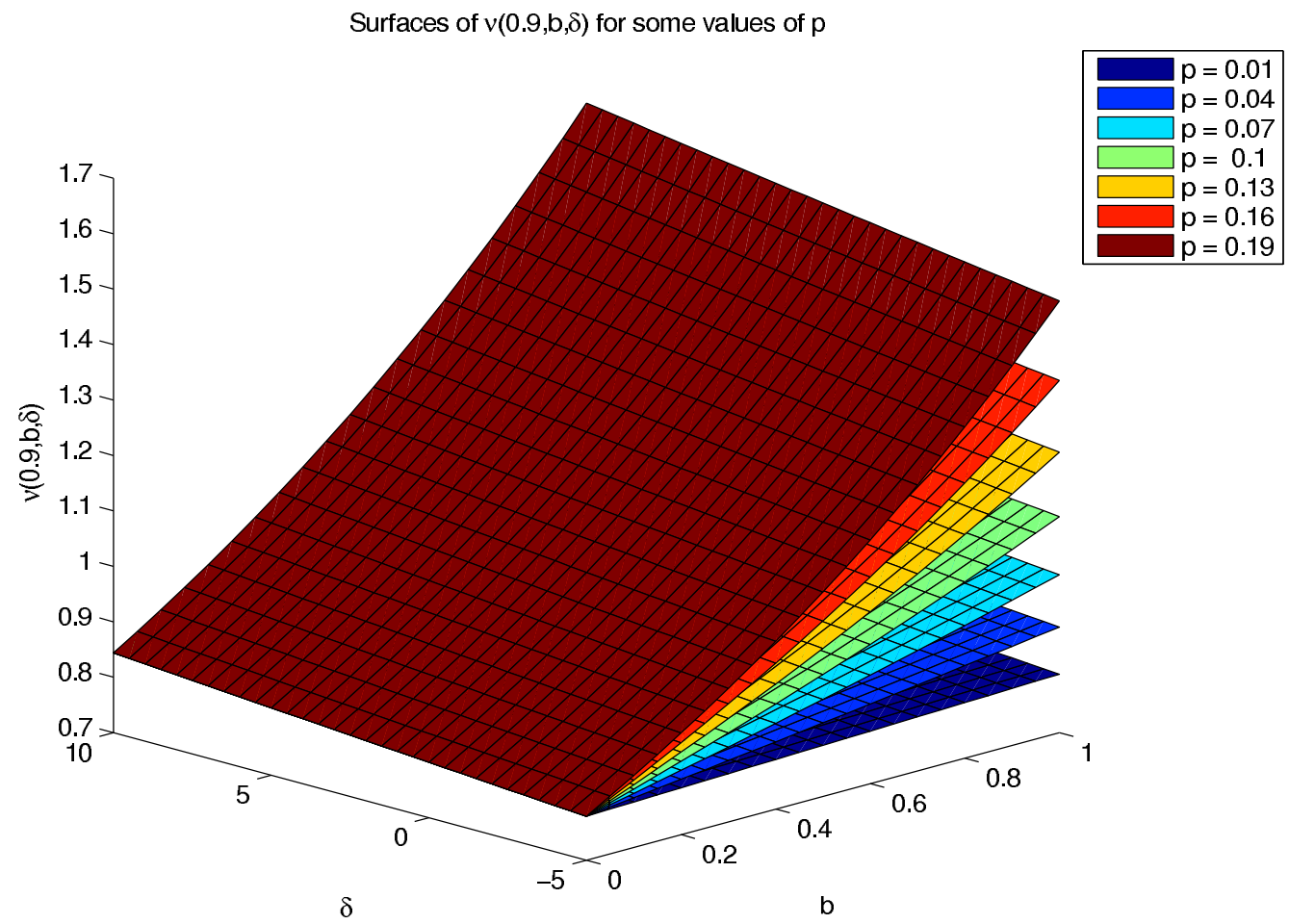

(b) Surface of $\nu(0.9, b, \delta)$ with $p_{1}=0.7, p_{2}=0, p_{3}=0.3, d=u=0.5$

Figure 1: Some surfaces of $\nu(t, b, \delta)$ when $t=0.9$ 


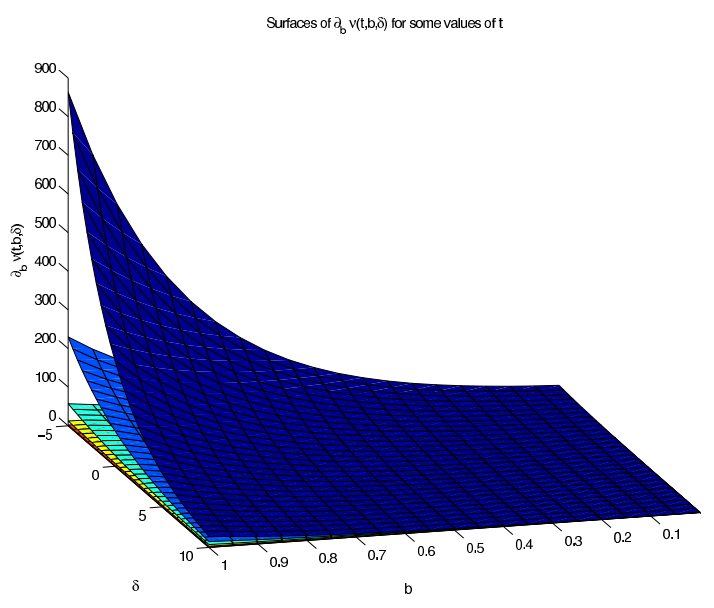

(a) The partial derivative $\partial_{b} \nu(t, b, \delta)$

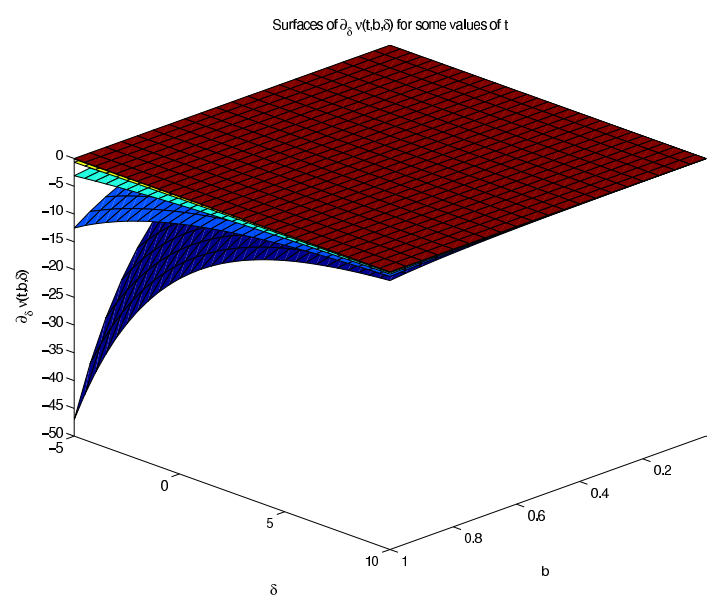

(b) The partial derivative $\partial_{\delta} \nu(t, b, \delta)$

Figure 2: Derivatives of $\nu(t, b, \delta)$ for some values of $t$

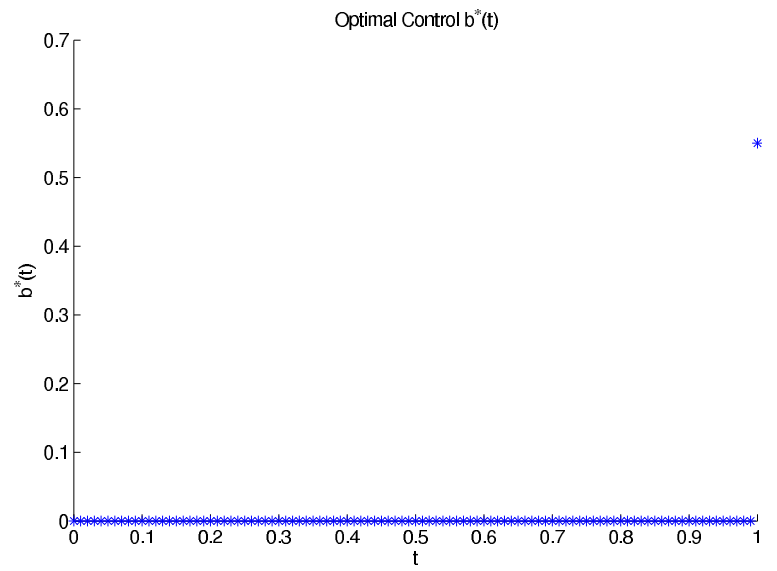

(a) Optimal control $b^{*}(t)$

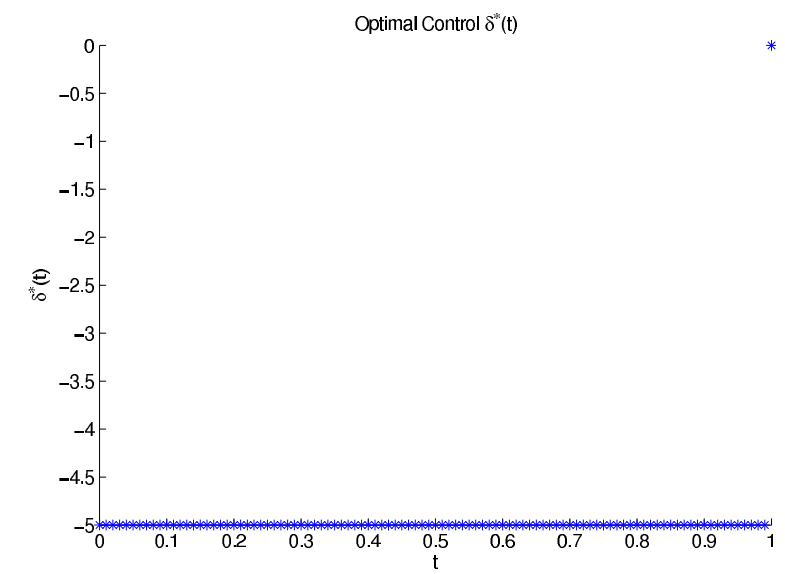

(b) Optimal control $\delta^{*}(t)$

Figure 3: Optimal controls $b^{*}(t)$ and $\delta^{*}(t)$ 


\subsection{Numerical iterative algorithm for general utility functions}

While for exponential utility functions, which allowed to obtain a solution that combines features of ruin minimization and expectation maximization, in the previous subsection 4.1 it was possible to obtain an explicit (semi)analytical solution, for general utility functions one has to look for an approximate solution by iterating the operator $T^{*}$ according to (17) and this approach is discussed in subsection 3.4. For this purpose one needs an efficient scheme to compute the iterates of $T^{*}$. It turns out that for this purpose the computationally most demanding part is the expectation in formula (13) of Definition 9. In the specific case of Example 13, which is the case considered in the calculations, this expectation becomes the integral in (19). A numerical quadrature formula for the computation of the integral leads to precise results but is time consuming so that a Monte Carlo (MC) approach appeared to be more convenient. Remaining within a reasonable number of samples of the MC approach, some numerical instability appeared for values of $t$ close to $T$ (this problem can be overcome by increasing sufficiently the number of samples). Using both a Matlab and Mathematica implementation of $T^{*}$, in Edoli(2009) a comparison is made between the exact results obtainable for exponential utilities (previous subsection 4.1.2) and those obtained on the basis of successive iterations of $T^{*}$. It turned out that up to values of $t$ not too close to $T$ the results are identical and lead to the optimal values. For values of $t$ very close to $T$ (and a reasonable number of MC samples) the computed controls differ slightly from the actual optimal ones because of the abovementioned numerical instability; again, this problem can be overcome by increasing sufficiently the number of MC samples.

Acknowledgements: Part of the contribution by the second author was obtained while he was visiting professor 2009 for the chair Quantitative Finance and Insurance at the LMU University in Munich funded by LMU Excellent. Hospitality and financial support are gratefully acknowledged.

\section{References}

[1] Bai,L., Guo, J., 2008. Optimal proportional reinsurance and investment with multiple risky assets and no-shorting constraint. Insurance: Mathematics and Economics 42, 968-975.

[2] Bäuerle,N., Rieder,U., 2009. MDP Algrotithms for Portfolio Optimization Problems in Pure Jump Markets. Finance and Stochastics 13, 591-611.

[3] Cao,Y., Wan,N., 2009. Optimal proportional reinsurance and investment based on HamiltonJacobi-Bellman equation. Insurance: Mathematics and Economics 45, 157-162.

[4] Di Masi,G.B., Callegaro,G., Runggaldier,W.J., 2006. Portfolio Optimization in Discontinuous Markets under Incomplete Information. Asia Pacific Financial Markets 13, 373-394.

[5] Edoli,E., 2009. On Optimal Investment in a Reinsurance Context with a Point Process Market Model. Master's Thesis, University of Padova.

[6] Hernandez-Lerma,O., Runggaldier,W.J., 1994. Monotone approximations for convex stochastic control problems. Journal of Mathematical Systems, Estimation, and Control 4, 99-140.

[7] Hipp,C., 2004. Stochastic Control with Application in Insurance. In: Frittelli,M., Runggaldier,W. (Eds.). Stochastic Methods in Finance. Lecture Notes in Mathematics,Vol.1856. Berlin/Heidelberg: Springer-Verlag, 127-164. 
[8] Kirch,M., Runggaldier,W.J., 2004. Efficien thedging when asset prices follow a geometric Poisson process with unknown intensities. SIAM J. on Control and Optimization 43, 11741195.

[9] Luo,S., Taksar,M., Tsoi,A., 2008. On reinsurance and investment for large insurance portfolios. Insurance: Mathematics and Economics 42, 434-444.

[10] Michael,E.A.,1970. A survey of continuous selections. Lecture Notes in Mathematics, Vol.171. Berlin/Heidelberg: Springer Verlag.

[11] Pham,H., Tankov,P., 2008. A model of optimal consumption under liquidity risk with random trading times. Mathematical Finance 18, 613-627.

[12] Pham,H., Tankov,P., 2009. A coupled system of integrodifferential equations arising in liquidity risk model. Applied Mathematics and Optimization 59, 147-173.

[13] Polyanin,A.D., Manzhirov,A.V., 2008. Handbook of Integral Equations, 2nd Edition. Chapman \& Hall/CRC Press.

[14] Schäl,M., 2004. On discrtete time dynamic programming in insurance: Exponential utility and minimizing the ruin probability. Scandinavian Actuarial Journal (3), 189-210.

[15] Schmidli,H., 2002. On minimizing the ruin probability by investment and reinsurance. The Annals of Applied Probability 12 (3), 890-907.

[16] Schmidli,H., 2008. Stochastic control in insurance. London: Springer Verlag. 